\title{
Realization of critical eigenvalues for scalar and symmetric linear delay-differential equations
}

\author{
P-L. Buono \\ Faculty of Science \\ University of Ontario Institute of Technology \\ Oshawa, ONT L1H 7K4 \\ Canada \\ V.G. LeBlanc \\ Department of Mathematics and Statistics \\ University of Ottawa \\ Ottawa, ONT K1N 6N5 \\ Canada
}

November 9, 2018

\begin{abstract}
This paper studies the link between the number of critical eigenvalues and the number of delays in certain classes of delay-differential equations. There are two main results. The first states that for $k$ purely imaginary numbers which are linearly independent over the rationals, there exists a scalar delay-differential equation depending on $k$ fixed delays whose spectrum contains those $k$ purely imaginary numbers. The second result is a generalization of the first result for delay-differential equations which admit a characteristic equation consisting of a product of $s$ factors of scalar type. In the second result, the $k$ eigenvalues can be distributed amongst the different factors. Since the characteristic equation of scalar equations contain only exponential terms, the proof exploits a toroidal structure which comes from the arguments of the exponential terms in the characteristic equation. Our second result is applied to delay coupled $\mathbf{D}_{n}$-symmetric cell systems with one-dimensional cells. In particular, we provide a general characterization of delay coupled $\mathbf{D}_{n}$-symmetric systems with arbitrary number of delays and cell dimension.
\end{abstract}

\section{Introduction and Background}

Delay-differential equations (DDEs) have been used as mathematical models for phenomena in population dynamics [20], physiology [12, 3], physics [22], climate modelling [28] and engineering [28] amongst others. Delay-differential equations behave like abstract ordinary differential equations (ODEs) on an infinite-dimensional (Banach) phase space and many results which are 
known for ODEs on finite-dimensional spaces have analogs in the context of DDEs. Many scalar delay-differential equation models have been developed over the years such as for Cheynes-Stokes respiration [12 and the regulation of hematopoiesis [12, the delayed Nicholson blowflies equation [17] in population dynamics, a two-delay model of an experiment on Parkinsonian tremor [2] and many more.

The bifurcation analysis of DDEs is done essentially in the same way as in ODEs, although the technical details differ. Consider the neighborhood of an equilibrium solution of a nonlinear DDE, then the analysis of the linearization at the equilibrium point leads to stable, unstable and centre invariant subspaces where only the stable subspace is infinite-dimensional. There exists local invariant manifolds (stable, unstable and center manifolds) tangent to the corresponding invariant subspaces of the linearized equations about the equilibrium point on which the flow near the equilibrium is either exponentially attracting (stable manifold), exponentially repelling (unstable manifold), or non-hyperbolic (center manifold). Now, bifurcations near equilibria are determined by the flow on the centre manifold and the dimension of this manifold is determined by the number of eigenvalues of the linearization on the imaginary axis.

The first result of our paper is Theorem 2.1 and goes as follows. Consider $n$ nonzero imaginary numbers $i \omega_{1}, \ldots, i \omega_{n}$ where the imaginary parts $\omega_{1}, \ldots, \omega_{n}$ are positive and not rationally dependent. We show that there exists a scalar linear delay-differential equation depending on $n$ discrete delays written

$$
\dot{x}=\sum_{j=1}^{n} a_{j} x\left(t-\tau_{j}\right)
$$

where $x \in \mathbb{R}, a_{j} \in \mathbb{R}$ and $\tau_{j} \in[0, \tau]$ for all $j=1, \ldots, n$ such that the characteristic equation of (1), given by

$$
\lambda-\sum_{j=1}^{n} a_{j} e^{\lambda \tau_{j}}=0,
$$

has eigenvalues $\pm i \omega_{1}, \ldots, \pm i \omega_{n}$. This result generalizes explicit computations done in the case of one and two delays, see [19, 9, 1,. The proof is done by embedding the problem as a mapping which is solved by the implicit function theorem at a carefully chosen point. From the implicit function theorem, we are able to define a smooth mapping whose transversal intersection with a dense curve on an $n$-dimensional torus provide solutions. The incommensurability of the $n$ frequencies enables us to define the dense curve on the $n$-torus. This type of argument using a dense curve on an $n$-dimensional torus was used in Choi and LeBlanc [7].

This result falls within the category of so-called realization theorems. For instance, the realization theorem of linear ODEs by linear DDEs obtained by Faria and Magalhães [11. They show that for any finite dimensional matrix $B$, a necessary and sufficient condition for the existence of a bounded linear operator $\mathcal{L}_{0}$ from $C\left([-\tau, 0], \mathbb{R}^{n}\right)$ into $\mathbb{R}^{n}$ with infinitesimal generator having spectrum containing the spectrum of $B$ is that $n$ be larger than or equal to the largest number of Jordan blocks associated with each eigenvalue of $B$. Other results in this direction are concerned with the realization of finite jets of ODEs on a finite-dimensional centre manifold by delay-differential equations, see [11, 7]. To our knowledge, the realization theorems in this paper 
are the first general results linking the number of critical eigenvalues of linear delay-differential equations with the number of discrete delays.

The next significant result is an openness theorem, that is, the realization of $n$ imaginary numbers (not necessarily rationally independent) as eigenvalues of a linear scalar delay-differential equation is valid in a neighborhood of any set of $n$ rationally independent imaginary numbers. The proof of this theorem also relies on the implicit function theorem.

We then turn our attention to the context of symmetric systems of delay-differential equations. Several examples of symmetric systems of DDEs [15, 24] have characteristic equations which decompose in factors, some of which have the same form as the characteristic equation (2). The decomposition of the characteristic equation is induced by the isotypic decomposition of the space and we present a general derivation of this decomposition. We show that isotypic components consisting of a unique one-dimensional complex irreducible representation contribute a factor of the form (2) in the characteristic equation and so Theorem 2.1 can be applied directly to each such factors separately.

We present a generalization of Theorem 2.1 to the case where several factors of the characteristic equation have purely imaginary eigenvalues simultaneously. Theorem 2.4 shows that a set of $n$ rationally independent purely imaginary complex numbers can be realized from several factors of the characteristic equation of a delay-differential equation with $n$ delays given some nondegeneracy conditions on the characteristic equation are satisfied. The statement of the theorem is independent of any symmetric structure and the proof is a generalization of the proof of Theorem 2.1.

We illustrate the above result on $\mathbf{D}_{n}$-symmetric rings of $n$ delay equations with delayed coupling. Hopf bifurcation from such symmetric networks have been studied by several authors [6, 14, 15, 16, 24, 25, 29]. In order to apply Theorem 2.4 to this context, we derive an explicit form of the coupling matrix in terms of the connections in the graph representation of the ring for cells of any dimension and arbitrary numbers of connections and delays. This is a generalization of the networks considered in the articles listed above in this paragraph.

We specialize to the case of one-dimensional cells and we can then obtain general formulae for the factors of the form (2) in the characteristic equation of the $\mathbf{D}_{n}$-symmetric ring. Given a nondegeneracy assumption of Theorem 2.4 is satisfied, then it can be applied directly to $\mathbf{D}_{n}$ symmetric coupled cell systems with $n$ odd. We do not treat the case $n$ even because the form of the equations needed for Theorem 2.4 is not satisfied. We illustrate this fact in a $\mathbf{D}_{4}$-symmetric example.

The paper is organized as follows. The first section contains brief preliminary remarks and then we state and prove our main result (Theorem 2.1) and the openness result (Theorem 2.2). Then we introduce the context leading to Theorem 2.4 and state this result. Section 3 is devoted to $\Gamma$ symmetric systems of delay-differential equations and the section begins with a general discussion. Section 3.1 presents a characterization of $\mathbf{D}_{n}$-symmetric rings of delay coupled cells with an arbitrary number of delays and derive the characteristic equation in the case of one-dimensional cells. In Section 3.2, Theorem 2.4 is applied to $\mathbf{D}_{n}$-symmetric rings of one-dimensional cells with $n$ odd. Section 4 has the proof of Theorem 2.4. We conclude by a discussion of open problems along the lines of the ones presented in this paper. 


\section{Realization theorems}

We now discuss some aspects of the spectral theory of linear scalar delay-differential equations. In fact, we just introduce the basic facts, in a non-abstract setting, needed for the statement of our first main theorem. For a complete treatment, see Diekmann et al [9] or Hale et Lunel [19].

Consider the scalar delay-differential equation

$$
\dot{x}(t)=\sum_{j=1}^{n} a_{j} x\left(t-\tau_{j}\right)
$$

where $a_{j} \in \mathbb{R}$ and $\tau_{j} \in[0, \tau]$ for all $j=1, \ldots, n$ and $\tau>0$. The characteristic equation for (3) can be obtained by substituting $x(t)=C e^{\lambda t}$, where $C$ is a constant, into the equation. Thus,

$$
\lambda C e^{\lambda t}=\sum_{j=1}^{n} a_{j} C e^{\lambda\left(t-\tau_{j}\right)}=\sum_{j=1}^{n} a_{j} C e^{-\lambda \tau_{j}} e^{\lambda t}
$$

and by rearranging the terms we obtain

$$
\left(\lambda-\sum_{j=1}^{n} a_{j} e^{-\lambda \tau_{j}}\right) x(t)=0 .
$$

So, $x(t)$ is a nonzero solution of (3) if and only if

$$
\Delta(\lambda):=\lambda-\sum_{j=1}^{n} a_{j} e^{-\lambda \tau_{j}}=0 .
$$

The complex number $\lambda$ is an eigenvalue of equation (3) if it is a solution of the characteristic equation $\Delta(\lambda)=0$.

The question we address in this paper is related to the number of imaginary eigenvalues (with incommensurable frequencies) which can satisfy $\Delta(\lambda)=0$. The case $n=1$ with one nonzero delay is a straightforward calculation and $\Delta(\lambda)=0$ for only one nonzero imaginary eigenvalue $\lambda$, see [19]. The case $n=2$ with $\tau_{1}=0$ and $\tau_{2} \in(0, \tau]$ in (3) can be found in [9]. There, it is shown that $\Delta(\lambda)=0$ can have at most two nonzero imaginary eigenvalues. The case $n=2$ with $\tau_{1}, \tau_{2}>0$ is done in [1] where it is shown that $\Delta(\lambda)=0$ can have at most two nonzero imaginary eigenvalues. We are now ready to state our first result.

Theorem 2.1 Suppose $\omega_{1}>0, \omega_{2}>0, \ldots, \omega_{n}>0$ are linearly independent over the rationals. Then there exists $\tau_{1}>0, \tau_{2}>0, \ldots, \tau_{n}>0, a_{1} \in \mathbb{R}, a_{2} \in \mathbb{R}, \ldots, a_{n} \in \mathbb{R}$ such that the linear delay differential equation

$$
\dot{x}(t)=a_{1} x\left(t-\tau_{1}\right)+a_{2} x\left(t-\tau_{2}\right)+\cdots+a_{n} x\left(t-\tau_{n}\right)
$$

has solutions $x_{j}^{ \pm}(t)=e^{ \pm i \omega_{j} t}$ for all $j=1, \ldots, n$. 
Proof: A necessary and sufficient condition for the conclusion of the theorem to hold is that the following algebraic system of $2 n$ equations

$$
\begin{aligned}
\sum_{k=1}^{n} a_{k} e^{-i \omega_{j} \tau_{k}} & =i \omega_{j}, \quad j=1, \ldots, n \\
\sum_{k=1}^{n} a_{k} e^{i \omega_{j} \tau_{k}} & =-i \omega_{j}, \quad j=1, \ldots, n
\end{aligned}
$$

has a solution in the $2 n$ unknowns $\left(\tau_{1}, \tau_{2}, \ldots, \tau_{n}, a_{1}, a_{2}, \ldots, a_{n}\right)$. Although (5) is in complex form, since the second equation in (5) is just the complex conjugate of the first equation in (5), system (5) is equivalent to a system of $2 n$ real equations. This fact is taken for granted throughout the sequel, even though we continue to use complex notation.

It is useful to use the following matrix notation for (5)

$$
\left(\begin{array}{c}
P(\tau ; \omega) \\
P(-\tau ; \omega)
\end{array}\right) A^{T}=\left(\begin{array}{c}
i \omega^{T} \\
-i \omega^{T}
\end{array}\right)
$$

where, $\omega=\left(\omega_{1}, \ldots, \omega_{n}\right), A=\left(a_{1}, \ldots, a_{n}\right)$, superscript $T$ denotes transpose, and $P(\tau ; \omega)=$ $P\left(\tau_{1}, \ldots, \tau_{n} ; \omega_{1}, \ldots, \omega_{n}\right)$ is the $n \times n$ matrix whose entry at row $j$ column $k$ is

$$
[P(\tau ; \omega)]_{j k}=e^{-i \omega_{j} \tau_{k}} .
$$

Note that $\overline{P(\tau ; \omega)}=P(-\tau ; \omega)$.

Remark: Let $\mathcal{P}$ denote the $2 n \times n$ matrix of coefficients of the left-hand side of (6)). If we view $\tau_{1} \in \mathbb{R}^{+}, \ldots, \tau_{n} \in \mathbb{R}^{+}$as free parameters for these coefficients, we see that each column of the matrix $\mathcal{P}$ is dense on an $n$-torus, $\mathrm{T} \equiv\left(\mathbb{S}^{1}\right)^{n}$, since the $\omega_{j}$ are rationally independent. This remark is a key point in the following discussion.

Define $\mathrm{V} \equiv \mathrm{T}^{n}=\left(\left(\mathbb{S}^{1}\right)^{n}\right)^{n}$, and choose coordinates on $\mathrm{V}$ as follows:

$$
\mathrm{V}=\left\{\Phi=\left(\Phi^{1}, \ldots, \Phi^{n}\right) \mid \Phi^{j}=\left(\varphi_{1}^{j}, \ldots, \varphi_{n}^{j}\right) \in\left(\mathbb{S}^{1}\right)^{n}, j=1, \ldots, n\right\} .
$$

Consider the following mapping associated to (6):

$$
F: \mathrm{V} \times \mathbb{R}^{n} \longmapsto \mathbb{R}^{2 n}
$$

defined by

$$
F(\Phi, A ; \omega)=\left(\begin{array}{c}
\widetilde{P}(\Phi) \\
\widetilde{P}(-\Phi)
\end{array}\right) A^{T}-i\left(\begin{array}{c}
\omega^{T} \\
-\omega^{T}
\end{array}\right),
$$

where $A$ and $\omega$ are as previously defined, and $\widetilde{P}(\Phi)$ is the $n \times n$ matrix whose entry at row $j$ column $k$ is

$$
[\widetilde{P}(\Phi)]_{j k}=\left[\widetilde{P}\left(\Phi^{1}, \ldots, \Phi^{n}\right)\right]_{j k}=e^{-i \varphi_{j}^{k}}
$$


For reasons that are clear from the sequel, we choose to single out the last torus in the product $\mathrm{V}=\mathrm{T}^{n}$, and use the variable $\Psi$ as coordinates for this torus, i.e.

$$
\Psi=\left(\psi_{1}, \ldots, \psi_{n}\right)=\Phi^{n}=\left(\varphi_{1}^{n}, \ldots, \varphi_{n}^{n}\right) .
$$

Thus, we write $\mathrm{V}=\mathrm{V}_{\Phi} \times \mathrm{V}_{\Psi}$, where $\mathrm{V}_{\Phi} \cong \mathrm{T}^{n-1}$ and $\mathrm{V}_{\Psi} \cong \mathrm{T}$, so that

$$
F: \mathrm{V}_{\Phi} \times \mathrm{V}_{\Psi} \times \mathbb{R}^{n} \longmapsto \mathbb{R}^{2 n}
$$

is written as $F(\Phi, \Psi, A ; \omega)$ in (7) (we have re-labeled $\Phi=\left(\Phi^{1}, \ldots, \Phi^{n-1}\right.$ ) to designate coordinates for $\left.V_{\Phi} \cong \mathrm{T}^{n-1}\right)$.

Definition: If $\left\{e_{1}, \ldots, e_{n}\right\}$ denotes the canonical basis of vectors in $\mathbb{R}^{n}$, we define the vectors $v_{1}, \ldots, v_{n}$ by $v_{1}=\sum_{k=1}^{n} e_{k}$, and for $j=2, \ldots, n$,

$$
v_{j}=v_{1}-\sum_{\ell=0}^{j-2} 2 e_{n-\ell} .
$$

By construction, the set $\left\{v_{1}, \ldots, v_{n}\right\}$ is linearly independent, and so the $n \times n$ matrix $\mathcal{I}$, whose $j^{\text {th }}$ column is the vector $v_{j}^{T}$, is invertible. We also define the $n \times n$ invertible matrix $\mathcal{U}_{j}$ to be the diagonal matrix whose $k^{\text {th }}$ diagonal element is the $k^{\text {th }}$ component of the vector $v_{j}$ (in particular, $\mathcal{U}_{1}$ is the identity matrix). Note also that $\mathcal{U}_{j}^{-1}=\mathcal{U}_{j}, j=1, \ldots, n$.

Consider the following point in $\mathrm{V}_{\Phi} \times \mathrm{V}_{\Psi}$ :

$$
(\widehat{\Phi}, \widehat{\Psi})=-\frac{\pi}{2}\left(\left(v_{1}, \ldots, v_{n-1}\right), v_{n}\right) ;
$$

then it is easy to compute that

$$
\widetilde{P}(\widehat{\Phi}, \widehat{\Psi})=i \mathcal{I}
$$

where $\widetilde{P}$ is as in (7). If we define

$$
\widehat{A}^{T} \equiv\left(\hat{a}_{1}, \ldots, \hat{a}_{n}\right)^{T}=\mathcal{I}^{-1} \omega^{T}
$$

then

$$
F(\widehat{\Phi}, \widehat{\Psi}, \widehat{A} ; \omega)=0 .
$$

Because the $\omega_{j}$ are rationally independent, it follows that the components $\hat{a}_{k}$ of $\widehat{A}$ are all non-zero. Furthermore, it is easy to compute the following derivatives

$$
J \equiv D_{(\Psi, A)} F(\widehat{\Phi}, \widehat{\Psi}, \widehat{A} ; \omega)=\left(\begin{array}{cc}
\hat{a}_{n} \mathcal{U}_{n} & i \mathcal{I} \\
\hat{a}_{n} \mathcal{U}_{n} & -i \mathcal{I}
\end{array}\right)
$$


and

$$
K \equiv D_{\Phi} F(\widehat{\Phi}, \widehat{\Psi}, \widehat{A} ; \omega)=\left(\begin{array}{cccc}
\hat{a}_{1} \mathcal{U}_{1} & \hat{a}_{2} \mathcal{U}_{2} & \cdots & \hat{a}_{n-1} \mathcal{U}_{n-1} \\
\hat{a}_{1} \mathcal{U}_{1} & \hat{a}_{2} \mathcal{U}_{2} & \cdots & \hat{a}_{n-1} \mathcal{U}_{n-1}
\end{array}\right)
$$

The matrix $J$ is invertible, since its inverse is easily computed as

$$
J^{-1}=\left(\begin{array}{cc}
\frac{1}{2 \hat{a}_{n}} \mathcal{U}_{n} & \frac{1}{2 \hat{a}_{n}} \mathcal{U}_{n} \\
-\frac{i}{2} \mathcal{I}^{-1} & \frac{i}{2} \mathcal{I}^{-1}
\end{array}\right) .
$$

By the implicit function theorem, there exists a neighborhood $N$ of $\widehat{\Phi}$ in $\mathrm{V}_{\Phi}$ and a unique smooth function

$$
\begin{aligned}
& G: N \quad \longmapsto \mathrm{V}_{\Psi} \times \mathbb{R}^{n} \\
& G: \Phi \quad \longmapsto G(\Phi)=\left(G_{\Psi}(\Phi), G_{A}(\Phi)\right)
\end{aligned}
$$

such that

$$
G(\widehat{\Phi})=(\widehat{\Psi}, \widehat{A})
$$

and

$$
F(\Phi, G(\Phi) ; \omega) \equiv 0, \forall \Phi \in N
$$

Implicit differentiation of (9) yields that

$$
\begin{aligned}
D G(\widehat{\Phi}) & =\left(\begin{array}{c}
D G_{\Psi}(\widehat{\Phi}) \\
D G_{A}(\widehat{\Phi})
\end{array}\right)=-J^{-1} K \\
& =\left(\begin{array}{cccc}
-\frac{\hat{a}_{1}}{\hat{a}_{n}} \mathcal{U}_{n} \mathcal{U}_{1} & -\frac{\hat{a}_{2}}{\hat{a}_{n}} \mathcal{U}_{n} \mathcal{U}_{2} & \cdots & -\frac{\hat{a}_{n-1}}{\hat{a}_{n}} \mathcal{U}_{n} \mathcal{U}_{n-1} \\
\mathbf{0} & \mathbf{0} & \cdots & \mathbf{0}
\end{array}\right),
\end{aligned}
$$

where $\mathbf{0}$ denotes the $n \times n$ zero matrix. Consequently,

$$
D G_{\Psi}(\widehat{\Phi})=\left(\begin{array}{llll}
-\frac{\hat{a}_{1}}{\hat{a}_{n}} \mathcal{U}_{n} \mathcal{U}_{1} & -\frac{\hat{a}_{2}}{\hat{a}_{n}} \mathcal{U}_{n} \mathcal{U}_{2} & \cdots & -\frac{\hat{a}_{n-1}}{\hat{a}_{n}} \mathcal{U}_{n} \mathcal{U}_{n-1}
\end{array}\right),
$$

and it follows that the mapping

$$
G_{\Psi}: N \longrightarrow \mathrm{V}_{\Psi}
$$

is regular at $\widehat{\Phi}$.

Consider the following $n-1$ vectors in $\left(\mathbb{R}^{n}\right)^{n-1}$ :

$$
\begin{aligned}
W_{1} & =(\omega, 0, \ldots, 0,0) \\
W_{2} & =(0, \omega, 0, \ldots, 0,0) \\
& \vdots \\
W_{n-2} & =(0,0, \ldots, \omega, 0) \\
W_{n-1} & =(0,0, \ldots, 0, \omega),
\end{aligned}
$$


where 0 represents the 0 vector in $\mathbb{R}^{n}$, and we recall that $\omega=\left(\omega_{1}, \ldots, \omega_{n}\right)$.

The set $\left\{W_{1}, \ldots, W_{n-1}\right\}$ is linearly independent, so for any $\Phi \in \mathrm{V}_{\Phi}$, the set

$$
\mathcal{S}_{\Phi}=\left\{\Phi+\sum_{j=1}^{n-1} \sigma_{j} W_{j}\left(\bmod \mathrm{V}_{\Phi}\right)|0 \leq| \sigma_{j} \mid<<1, j=1, \ldots, n\right\}
$$

is a small $n$-1-dimensional surface through $\Phi$ in $\mathrm{V}_{\Phi}$. We are interested in showing that for $\Phi$ close enough to $\widehat{\Phi}$ in $N$, the image of $\mathcal{S}_{\Phi}$ by $G_{\Psi}$ in $\mathrm{V}_{\Psi}$ is transverse to the integral curves of the vector field $\dot{\Psi}=\omega$. To show this, we consider the function

$$
\mathcal{T}: N \longrightarrow \mathbb{R}
$$

defined by

$$
\mathcal{T}(\Phi)=\operatorname{det}\left(D G_{\Psi}(\Phi) \cdot W_{1}^{T} \quad D G_{\Psi}(\Phi) \cdot W_{2}^{T} \quad \cdots \quad D G_{\Psi}(\Phi) \cdot W_{n-1}^{T} \quad \omega^{T}\right) .
$$

Obviously, $\mathcal{T}$ is continuous, and

$$
\begin{aligned}
\mathcal{T}(\widehat{\Phi}) & =\operatorname{det}\left(\begin{array}{lllll}
-\frac{\hat{a}_{1}}{\hat{a}_{n}} \mathcal{U}_{n} \mathcal{U}_{1} \omega^{T} & -\frac{\hat{a}_{2}}{\hat{a}_{n}} \mathcal{U}_{n} \mathcal{U}_{2} \omega^{T} & \cdots & -\frac{\hat{a}_{n-1}}{\hat{a}_{n}} \mathcal{U}_{n} \mathcal{U}_{n-1} \omega^{T} & \mathcal{U}_{n} \mathcal{U}_{n} \omega^{T}
\end{array}\right) \\
& =\frac{(-1)^{n-1}}{\hat{a}_{n}^{n-1}} \operatorname{det} \mathcal{U}_{n} \operatorname{det}\left(\begin{array}{lllll}
\hat{a}_{1} \mathcal{U}_{1} \omega^{T} & \hat{a}_{2} \mathcal{U}_{2} \omega^{T} & \cdots & \hat{a}_{n-1} \mathcal{U}_{n-1} \omega^{T} & \mathcal{U}_{n} \omega^{T}
\end{array}\right) \\
& =\frac{\left(\omega_{1} \omega_{2} \cdots \omega_{n}\right)\left(\hat{a}_{1} \hat{a}_{2} \cdots \hat{a}_{n-1}\right)}{\hat{a}_{n}^{n-1}} \operatorname{det} \mathcal{I} \\
& \neq 0 .
\end{aligned}
$$

It follows that there is a neighborhood $N^{\prime} \subseteq N$ in which $\mathcal{T} \neq 0$. This is equivalent to saying that for all $\Phi \in N^{\prime}$, the image of $\mathcal{S}_{\Phi}$ by $G_{\Psi}$ in $\mathrm{V}_{\Psi}$ is transverse to the integral curves of the vector field $\dot{\Psi}=\omega$.

For each $j=1, \ldots, n-1$, the integral curves of the vector field $\dot{\Phi}^{j}=\omega$ are dense. Thus, for any $\varepsilon>0$, there is a $\tau_{j, \varepsilon}>0$ and an $s_{j, \varepsilon}>0$ such that the integral curve segment

$$
\left\{\Phi^{j}=\tau_{j} \omega\left(\bmod \left(\mathbb{S}^{1}\right)^{n}\right) \mid \tau_{j, \varepsilon}-s_{j, \varepsilon}<\tau_{j}<\tau_{j, \varepsilon}+s_{j, \varepsilon}\right\}
$$

is in the $\varepsilon$-ball centered on $\frac{\pi}{2} v_{j}$ in $\left(\mathbb{S}^{1}\right)^{n}$. For $\varepsilon>0$ small enough, the surface

$$
\left\{\Phi=\left(\tau_{1} \omega, \tau_{2} \omega, \ldots, \tau_{n-1} \omega\right)\left(\bmod \mathrm{V}_{\Phi}\right) \mid \tau_{j, \varepsilon}-s_{j, \varepsilon}<\tau_{j}<\tau_{j, \varepsilon}+s_{j, \varepsilon}\right\}
$$

is contained in $N^{\prime}$ and coincides with the surface $\mathcal{S}_{\Phi^{*}}$ for

$$
\Phi^{*}=\left(\tau_{1, \varepsilon} \omega, \tau_{2, \varepsilon} \omega, \ldots, \tau_{(n-1), \varepsilon} \omega\right)\left(\bmod \mathrm{V}_{\Phi}\right) .
$$

Thus, by our previous result, the $n$-1-dimensional surface $G_{\Psi}\left(\mathcal{S}_{\Phi^{*}}\right)$ is transverse to the integral curves of $\dot{\Psi}=\omega$ in $\mathrm{V}_{\Psi}$. Since these integral curves are dense in $\mathrm{V}_{\Psi}$, there are infinitely many intersections with $G_{\Psi}\left(\mathcal{S}_{\Phi^{*}}\right)$ near the point $\widehat{\Psi}=G_{\Psi}(\widehat{\Phi})$. 
Let $\stackrel{\circ}{\Psi} \in G_{\Psi}\left(\mathcal{S}_{\Phi^{*}}\right)$ be such an intersection point near $\widehat{\Psi}$. Then there is a $\stackrel{\circ}{\tau}_{n}>0$ such that

$$
\stackrel{\circ}{\Psi}=\stackrel{\circ}{\tau_{n}} \omega\left(\bmod \mathrm{V}_{\Psi}\right) \text {. }
$$

Let $\stackrel{\circ}{\Phi} \in \mathcal{S}_{\Phi^{*}}$ be such that $G_{\Psi}(\stackrel{\circ}{\Phi})=\stackrel{\circ}{\Psi}$. Then there are $\stackrel{\circ}{\tau}_{1}>0, \stackrel{\circ}{\tau}_{2}>0, \ldots, \stackrel{\circ}{\tau}_{n-1}>0$ such that

$$
\stackrel{\circ}{\Phi}=\left(\stackrel{\circ}{\tau}_{1} \omega, \stackrel{\circ}{\tau}_{2} \omega, \ldots, \stackrel{\circ}{\tau}_{n-1} \omega\right)\left(\bmod \mathrm{V}_{\Phi}\right) .
$$

It follows from (9) that $F\left(\stackrel{\circ}{\Phi}, \stackrel{\circ}{\Psi}, G_{A}(\stackrel{\circ}{\Phi}) ; \omega\right)=0$, and by construction, this corresponds to a solution of (므).

The next theorem shows that the previous realization result holds for open sets near solutions found in Theorem 2.1.

Theorem 2.2 Suppose $\omega_{1}>0, \omega_{2}>0, \ldots, \omega_{n}>0$ are linearly independent over the rationals. There exists a neigborhood $\mathcal{N}$ of $\omega=\left(\omega_{1}, \ldots, \omega_{n}\right)$ in $\mathbb{R}^{n}$ and a smooth mapping

$$
\begin{aligned}
H: V & \longrightarrow \mathbb{R}^{n} \times \mathbb{R}^{n} \\
\omega & \longmapsto H(\omega)=(\tau(\omega), A(\omega))=\left(\left(\tau_{1}(\omega), \ldots, \tau_{n}(\omega)\right),\left(a_{1}(\omega), \ldots, a_{n}(\omega)\right)\right)
\end{aligned}
$$

such that

for all $\omega \in \mathcal{N}$.

$$
\begin{aligned}
\sum_{k=1}^{n} a_{k}(\omega) e^{-i \omega_{j} \tau_{k}(\omega)} & =i \omega_{j}, \quad j=1, \ldots, n \\
\sum_{k=1}^{n} a_{k}(\omega) e^{i \omega_{j} \tau_{k}(\omega)} & =-i \omega_{j}, \quad j=1, \ldots, n
\end{aligned}
$$

Proof: We consider the system $F=0$ given by (7). We have already shown in Theorem 2.1 that, for fixed $\omega$ linearly independent over the rationals, there exists infinitely many solutions to $F=0$. We again use an implicit function theorem argument combined with the density of irrational torus flows.

Consider the mapping

$$
\begin{aligned}
Q: \mathbb{R}^{n} \times \mathbb{R}^{n} \times \mathbb{R}^{n} & \longrightarrow \mathbb{R}^{n} \times \mathbb{R}^{n} \\
(\tau, A, \omega) & \longmapsto Q(\tau, A, \omega)=F\left(\left(\tau_{1} \omega, \ldots, \tau_{n-1} \omega\right), \tau_{n} \omega, A ; \omega\right),
\end{aligned}
$$

where $F$ is as in (7). Therefore

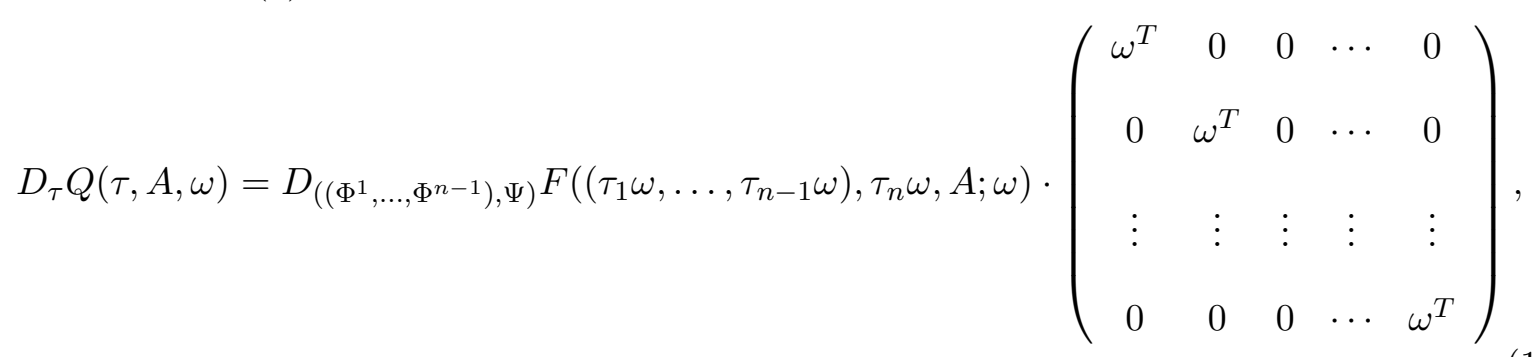


where each 0 in the matrix above is an $n$-dimensional zero column vector; and

$$
D_{A} Q(\tau, A, \omega)=D_{A} F\left(\left(\tau_{1} \omega, \ldots, \tau_{n-1} \omega\right), \tau_{n} \omega, A ; \omega\right)
$$

Thus, we wish to show that the $2 n \times 2 n$ matrix

$$
\left(D_{\tau} Q(\tau, A, \omega) \quad D_{A} Q(\tau, A, \omega)\right)
$$

is invertible at the solutions to (5) we have found in Theorem 2.1,

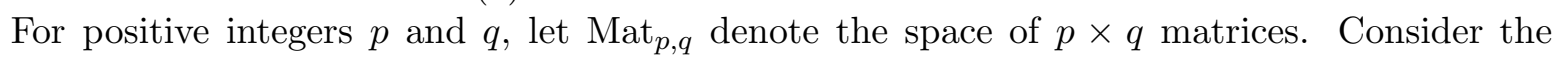
following mappings associated to (15), (16) and (17):

$$
\mathcal{R}_{1}: \mathrm{V}_{\Phi} \times \mathrm{V}_{\Psi} \times \mathbb{R}^{n} \times \mathbb{R}^{n} \longrightarrow \operatorname{Mat}_{2 n, n}
$$

defined by

$$
\mathcal{R}_{1}(\Phi, \Psi, A, \omega)=D_{(\Phi, \Psi)} F(\Phi, \Psi, A ; \omega) \cdot\left(\begin{array}{ccccc}
\omega^{T} & 0 & 0 & \cdots & 0 \\
& \mathcal{R}_{2}: \mathrm{V}_{\Psi} \times \mathrm{V}_{\Psi} \times \mathbb{R}^{n} \times \mathbb{R}^{n} \longrightarrow & 0 & \cdots & 0 \\
\vdots & \vdots & \vdots & \vdots & \vdots \\
0 & 0 & 0 & \cdots & \omega^{T}
\end{array}\right),
$$

defined by

$$
\mathcal{R}_{2}(\Phi, \Psi, A, \omega)=D_{A} F(\Phi, \Psi, A ; \omega)
$$

and

$$
\mathcal{R}: \mathrm{V}_{\Phi} \times \mathrm{V}_{\Psi} \times \mathbb{R}^{n} \times \mathbb{R}^{n} \longrightarrow \operatorname{Mat}_{2 n, 2 n}
$$

defined by

$$
\mathcal{R}(\Phi, \Psi, A, \omega)=\left(\mathcal{R}_{1}(\Phi, \Psi, A, \omega) \quad \mathcal{R}_{2}(\Phi, \Psi, A, \omega)\right) .
$$

Now, a simple computation (similar to those done in the proof of Theorem 2.1) shows that

$$
\mathcal{R}\left(-\frac{\pi}{2}\left(v_{1}, \ldots, v_{n}\right), A, \omega\right)=\left(\begin{array}{cc}
\mathcal{Z} & i \mathcal{I} \\
\mathcal{Z} & -i \mathcal{I}
\end{array}\right)
$$

where

$$
\mathcal{Z}=\left(\begin{array}{llll}
a_{1} \mathcal{U}_{1} \omega^{T} & a_{2} \mathcal{U}_{2} \omega^{T} & \cdots & a_{n} \mathcal{U}_{n} \omega^{T}
\end{array}\right) .
$$

If none of the $a_{j}$ vanish, then the $n \times n$ matrix $\mathcal{Z}$ is invertible, since its determinant is

$$
\operatorname{det} \mathcal{Z}=\prod_{j=1}^{n} a_{j} \omega_{j} \operatorname{det} \mathcal{I} \neq 0 .
$$


Thus,

$$
\mathcal{R}\left(-\frac{\pi}{2}\left(v_{1}, \ldots, v_{n}\right), A, \omega\right)^{-1}=\left(\begin{array}{cc}
\frac{1}{2} \mathcal{Z}^{-1} & \frac{1}{2} \mathcal{Z}^{-1} \\
-\frac{i}{2} \mathcal{I}^{-1} & \frac{i}{2} \mathcal{I}^{-1}
\end{array}\right)
$$

By continuity, there is thus a neighborhood $\mathcal{N}$ of the point $-\frac{\pi}{2}\left(v_{1}, \ldots, v_{n}\right)$ in $\mathrm{V}_{\Phi} \times \mathrm{V}_{\Psi}$ in which $\mathcal{R}$ is invertible. By Theorem 2.1, there are infinitely many solutions of $Q=0$ (see (14)) in $\mathcal{N}$, and the Jacobian matrix (17) is thus invertible at these solutions. We get the conclusion of Theorem 2.2 by the implicit function theorem.

\subsection{Example: $\mathrm{D}_{3}$-symmetric system}

Theorem 2.1 is written in the context of scalar delay-differential equations. However, in this section, we look at an example of a $\mathbf{D}_{3}$-symmetric system of delay-differential equations where Theorem 2.1 can be applied and then proceed to explain the generalization of this theorem which has applications to symmetric systems of delay-differential equations.

Example 2.3 Let $\Gamma=\mathbf{D}_{3}$ be the group generated by $\kappa$ and $\gamma$ act on $\mathbb{R}^{3}$ as follows:

$$
\kappa \cdot\left(x_{1}, x_{2}, x_{3}\right)=\left(x_{1}, x_{3}, x_{2}\right), \quad \gamma \cdot\left(x_{1}, x_{2}, x_{3}\right)=\left(x_{3}, x_{1}, x_{2}\right) .
$$

Consider a linear $\mathbf{D}_{3}$-symmetric coupled cell system with delayed coupling where each cell is one-dimensional and has the following form.

$$
\begin{aligned}
& \dot{x}_{1}=a_{1} x_{1}\left(t-\tau_{1}\right)+a_{2}\left[x_{2}\left(t-\tau_{2}\right)+x_{3}\left(t-\tau_{2}\right)\right] \\
& \dot{x}_{2}=a_{1} x_{2}\left(t-\tau_{1}\right)+a_{2}\left[x_{3}\left(t-\tau_{2}\right)+x_{1}\left(t-\tau_{2}\right)\right] \\
& \dot{x}_{3}=a_{1} x_{3}\left(t-\tau_{1}\right)+a_{2}\left[x_{1}\left(t-\tau_{2}\right)+x_{2}\left(t-\tau_{2}\right)\right] .
\end{aligned}
$$

where $x_{i} \in \mathbb{R}$ for $i=1,2,3$ and $a_{1}, a_{2}, a_{3} \in \mathbb{R}$. The characteristic equation of system (18) is obtained by substituting $\left(x_{1}, x_{2}, x_{3}\right)=\left(w_{1} e^{\lambda t}, w_{2} e^{\lambda t}, w_{3} e^{\lambda t}\right)$ into the equations. We obtain after simplification

$$
\begin{aligned}
& \lambda w_{1}=a_{1} e^{-\lambda \tau_{1}} w_{1}+a_{2} e^{-\lambda \tau_{2}}\left[w_{2}+w_{3}\right] \\
& \lambda w_{2}=a_{1} e^{-\lambda \tau_{1}} w_{2}+a_{2} e^{-\lambda \tau_{2}}\left[w_{3}+w_{1}\right] \\
& \lambda w_{3}=a_{1} e^{-\lambda \tau_{1}} w_{3}+a_{2} e^{-\lambda \tau_{2}}\left[w_{1}+w_{2}\right]
\end{aligned}
$$

and rearranging the terms we have

$$
\left[\left(\lambda-a_{1} e^{-\lambda \tau_{1}}\right) I-a_{2} e^{-\lambda \tau_{2}}\left(\begin{array}{ccc}
0 & 1 & 1 \\
1 & 0 & 1 \\
1 & 1 & 0
\end{array}\right)\right]\left(\begin{array}{c}
w_{1} \\
w_{2} \\
w_{3}
\end{array}\right)=0
$$

where $I$ is the $3 \times 3$ identity matrix. Letting $\alpha=\lambda-a_{1} e^{-\lambda \tau_{1}}$ and $\beta=-a_{2} e^{-\lambda \tau_{2}}$ equation (19) becomes

$$
\left(\begin{array}{lll}
\alpha & \beta & \beta \\
\beta & \alpha & \beta \\
\beta & \beta & \alpha
\end{array}\right)\left(\begin{array}{l}
w_{1} \\
w_{2} \\
w_{3}
\end{array}\right)=0
$$


Let

$$
\Delta(\lambda)=\left(\begin{array}{lll}
\alpha & \beta & \beta \\
\beta & \alpha & \beta \\
\beta & \beta & \alpha
\end{array}\right) .
$$

We complexify $\mathbb{R}^{3}$ and look at the isotypic decomposition of $\mathbb{C}^{3}$ by the action of $\mathbf{D}_{3}$ :

$$
\mathbb{C}^{3}=V_{0} \oplus V_{1} \oplus V_{2}
$$

where $V_{0}$ is the trivial representation of $\mathbf{D}_{3}$ and $V_{1}, V_{2}$ are the standard irreducible representations of $\mathbf{D}_{3}$ (all representations are one-dimensional complex). A basis for $V_{0}$ is $u_{0}=(v, v, v)^{t}$, a basis for $V_{1}$ is $u_{1}=\left(v, e^{2 \pi i / 3} v, e^{4 \pi i / 3} v\right)$ and a basis for $V_{2}$ is $u_{2}=\left(v, e^{4 \pi i / 3} v, e^{2 \pi i / 3} v\right)$. Therefore,

$$
\Delta(\lambda) u_{0}=(\alpha+2 \beta) u_{0}
$$

and

$$
\Delta(\lambda) u_{1}=(\alpha-\beta) u_{1}, \quad \Delta(\lambda) u_{2}=(\alpha-\beta) u_{2}
$$

since $e^{4 \pi i / 3}=\overline{e^{2 \pi i / 3}}$. Therefore, in the basis given by the isotypic decomposition of $\mathbb{C}^{3}, \Delta(\lambda)$ block diagonalizes so that we have

$$
\left(\begin{array}{ccc}
\alpha+2 \beta & 0 & 0 \\
0 & \alpha-\beta & 0 \\
0 & 0 & \alpha-\beta
\end{array}\right)\left(\begin{array}{c}
\tilde{w}_{1} \\
\tilde{w}_{2} \\
\tilde{w}_{3}
\end{array}\right)=0 .
$$

Hence, the eigenvalues are solutions to

$$
\operatorname{det} \Delta(\lambda)=(\alpha+2 \beta)(\alpha-\beta)^{2}=\left(\lambda-a_{1} e^{-\lambda \tau_{1}}-2 a_{2} e^{-\lambda \tau_{2}}\right)\left(\lambda-a_{1} e^{-\lambda \tau_{1}}+a_{2} e^{-\lambda \tau_{2}}\right)^{2}=0 .
$$

Each factor of the characteristic equation is of the same form as the characteristic equation for a scalar delay-differential equation. Therefore, by letting $\tilde{a}_{1}=a_{1}$ and $\tilde{a}_{2}=2 a_{2}$ in $\left(\lambda-a_{1} e^{-\lambda \tau_{1}}\right.$ $\left.2 a_{2} e^{-\lambda \tau_{2}}\right)$, Theorem 2.1 applies directly. The same is true for the factor $\left(\lambda-a_{1} e^{-\lambda \tau_{1}}+a_{2} e^{-\lambda \tau_{2}}\right)$ where we let $\tilde{a}_{1}=a_{1}$ and $\tilde{a}_{2}=-a_{2}$. Hence, for any choice of a set of complex numbers $\Lambda=$ $\left\{i \omega_{1}, i \omega_{2}\right\}$ with $\omega_{1}, \omega_{2}>0$ and rationally independent, there exists a linear $\mathbf{D}_{3}$ symmetric coupled cell system including $\Lambda$ in its spectrum.

In the context of bifurcation theory, the symmetry properties of the critical eigenspace depends on which factor contains the critical eigenvalue and this leads to different bifurcation behaviour. Two imaginary eigenvalues in the first factor corresponds to a nonresonant Hopf/Hopf mode interaction (without symmetry) while the second case leads to a nonresonant $\mathbf{D}_{3}$ Hopf/Hopf mode interaction. Details of the unfolding of these bifurcations can be found respectively in Kuznetsov [21] and Golubitsky et. al. [13].

Note that Theorem 2.1 is not sufficient to guarantee the existence of a linear $\mathbf{D}_{3}$ symmetric coupled cell system with $i \omega_{1}$ satisfying the first factor and $i \omega_{2}$ satisfying the second factor simultaneously. We characterize this situation as follows. Let $b_{1}^{1}=b_{1}^{2}=1$ and $b_{2}^{1}=2$ and $b_{2}^{2}=-1$ and for fixed rationally independent $i \omega_{1}, i \omega_{2}$ (with $\omega_{1}, \omega_{2}>0$ ), we look for $a_{1}, a_{2}$ and $\tau_{1}, \tau_{2}$ such that

$$
\begin{aligned}
& a_{1} b_{1}^{1} e^{-i \omega_{1} \tau_{1}}+a_{2} b_{2}^{1} e^{-i \omega_{1} \tau_{2}}=i \omega_{1} \\
& a_{1} b_{1}^{2} e^{-i \omega_{2} \tau_{1}}+a_{2} b_{2}^{2} e^{-i \omega_{2} \tau_{2}}=i \omega_{2}
\end{aligned}
$$


and their complex conjugate equations are satisfied. This is the context of the next theorem which is a generalization of Theorem 2.1. We state this result in a general form below and postpone the proof to Section 4 as it follows similar steps as the proof of Theorem 2.1.

Note that in the proof of Theorem 2.1, the matrix $\mathcal{I}$ defined in (8) is nonsingular by construction and this is a crucial step in the argument. For this more general result we shall present, the matrix which holds a similar role is denoted by $\mathcal{I}_{B}$ since it is a matrix consisting of \pm the constants $b_{k}^{j}$ which appear in equations (20). The form of this matrix is not relevant for the moment and the structure of the matrix is described in Section 4. We are now ready to state the theorem.

Theorem 2.4 Consider the factors

$$
\prod_{j=1}^{r}\left(\lambda-\sum_{k=1}^{n} a_{k} b_{k}^{j} e^{\lambda \tau_{k}}\right)
$$

of a characteristic polynomial where the constants $b_{k}^{j} \in \mathbb{R} \backslash\{0\}$ are fixed for all $j=1, \ldots, r$, $k=1, \ldots, n$ and suppose that $\operatorname{det} \mathcal{I}_{B} \neq 0$. Suppose that $\omega_{1}>0, \omega_{2}>0, \ldots, \omega_{n}>0$ are linearly independent over the rationals. Then there exists $\tau_{1}>0, \tau_{2}>0, \ldots, \tau_{n}>0, a_{1} \in \mathbb{R}, a_{2} \in$ $\mathbb{R}, \ldots, a_{n} \in \mathbb{R}$ such that for all $j=1, \ldots, r$,

$$
\left(\lambda-\sum_{k=1}^{n} a_{k} b_{k}^{j} e^{\lambda \tau_{k}}\right)=0
$$

has roots $i \omega_{\ell}^{j}$ for $\ell=1, \ldots, \ell_{j}$ where $\ell_{1}+\cdots+\ell_{r}=n$.

This theorem is applied in the following sections to the case of $\mathbf{D}_{n}$ symmetric coupled onedimensional cell systems. If $n$ odd, it is easy to show that $b_{k}^{j} \neq 0$ holds, but for $n$ even, several of the $b_{k}^{j}$ 's can be zero and Theorem 2.4 cannot be applied directly.

\section{Linear $\Gamma$-symmetric delay-differential equations}

For the results of this section, we find it convenient to introduce the well know abstract setting, see for instance Hale and Lunel [19], adapted to the symmetric case. Let $C_{n}=C\left([-\tau, 0], \mathbb{C}^{n}\right)$ be the Banach space of continuous functions from the interval $[-\tau, 0]$, into $\mathbb{C}^{n}(\tau>0)$ endowed with the norm of uniform convergence. Consider the linear homogeneous RFDE

$$
\dot{z}(t)=\mathcal{L}_{0}\left(z_{t}\right)
$$

where $\mathcal{L}_{0}$ is a bounded linear operator from $C_{n}$ into $\mathbb{C}^{n}$. We write

$$
\mathcal{L}_{0}(\varphi)=\int_{-\tau}^{0} d \eta(\theta) \varphi(\theta)
$$


where $\eta$ is an $n \times n$ matrix-valued function of bounded variation defined on $[-\tau, 0]$. The characteristic equation is

$$
\operatorname{det} \Delta(\lambda)=0, \quad \text { where } \Delta(\lambda)=\lambda I_{n}-\int_{-\tau}^{0} d \eta(\theta) e^{\lambda \theta},
$$

where $I_{n}$ is the $n \times n$ identity matrix. Note that $e^{\lambda \theta}=e^{\lambda \theta} I_{n}$.

Suppose that $\Gamma$ is a compact group of transformations acting linearly on $\mathbb{C}^{n}$. We say that (22) is $\Gamma$-equivariant if

$$
\gamma \cdot \eta(\theta)=\eta(\theta) \cdot \gamma, \forall \gamma \in \Gamma, \theta \in[-\tau, 0] .
$$

The group action of $\Gamma$ on $\mathbb{C}^{n}$ induces an isotypic decomposition of $\mathbb{C}^{n}$ :

$$
\mathbb{C}^{n}=V_{1} \oplus V_{2} \oplus \cdots V_{k}
$$

where $V_{i}=U_{i} \oplus \cdots U_{i}$ for irreducible representations $U_{i}$ of $\Gamma$ and $U_{i} \not U_{j}$ for $i \neq j$. Since $\eta(\theta)$ commutes with the action of $\Gamma$, then

$$
\eta(\theta) V_{i} \subset V_{i}
$$

for all $i=1, \ldots, k$.

Therefore, $\Delta(\lambda)$ also commutes with the representation of $\Gamma$. Indeed, for all $\gamma \in \Gamma$

$$
\begin{aligned}
\Delta(\lambda) \gamma & =\lambda I \gamma-\left[\int_{-\tau}^{0} d \eta(\theta) e^{\lambda \theta}\right] \gamma \\
& =\gamma \lambda I-\left[\int_{-\tau}^{0} d \eta(\theta) \gamma e^{\lambda \theta}\right] \\
& =\gamma \lambda I-\left[\int_{-\tau}^{0} \gamma d \eta(\theta) e^{\lambda \theta}\right] \\
& =\gamma\left(\lambda I-\int_{-\tau}^{0} d \eta(\theta) e^{\lambda \theta}\right)=\gamma \Delta(\lambda) .
\end{aligned}
$$

Thus,

$$
\Delta(\lambda) V_{i} \subset V_{i}
$$

and for all $i=1, \ldots, k$ and we can write $\Delta(\lambda)$ in block diagonal form:

$$
\Delta(\lambda)=\operatorname{diag}\left(\Delta_{1}(\lambda), \ldots, \Delta_{k}(\lambda)\right) .
$$

The characteristic equation then becomes

$$
\operatorname{det} \Delta(\lambda)=\prod_{i=1}^{k} \operatorname{det} \Delta_{i}(\lambda) .
$$

Therefore we are led to the following result.

Proposition 3.1 Suppose that $V_{i}=U_{i}$ and $U_{i}$ is a one-dimensional irreducible representation of Г. Then

$$
\operatorname{det} \Delta_{i}(\lambda)=\lambda-\sum_{j=1}^{\ell} a_{j} e^{\lambda \tau_{j}}
$$

Corollary 3.2 Theorem 2.1 applies to factors of the characteristic equation which correspond to the context of Proposition 3.1. 


\subsection{Delayed coupled cell systems with $\mathrm{D}_{n}$-symmetry}

Multiple authors have studied Hopf bifurcation in $\mathbf{D}_{n}$ symmetric rings of cells with delayed coupling where each cell is one-dimensional. The differential equation systems in those papers have the following general form. For $i=1, \ldots, n$, the dynamics of cell $i$ is given respectively for $n$ odd and $n$ even by:

$$
\begin{gathered}
\dot{x}_{i}(t)=f\left(X_{i}\right)+g\left(x_{i+1}, \ldots, x_{i+(n-1) / 2}, x_{i-(n-1) / 2}, \ldots, x_{i-1}\right) \\
\dot{x}_{i}(t)=f\left(X_{i}\right)+g\left(x_{i+1}, \ldots, x_{i+(n / 2-1)}, x_{i+n / 2}, x_{i-(n / 2-1)}, \ldots, x_{i-1}\right)
\end{gathered}
$$

where $X_{i}=\left(x_{i}\left(t-s_{1}\right), \ldots, x_{i}\left(t-s_{m}\right)\right), x_{j}=x_{j}\left(t-\tau_{j}\right)$ for $j \neq i, f: \mathbb{R}^{m} \rightarrow \mathbb{R}, g: \mathbb{R}^{n-1} \rightarrow \mathbb{R}$ are smooth functions and $\tau_{j}, s_{\ell} \in[0, \tau]$ for all $j \neq i$ and $s=1, \ldots, m$. Here, $f$ is called the internal dynamics and $g$ is the coupling function. In the papers described above, these systems are $\mathbf{D}_{n}$-equivariant.

\subsubsection{Characterization of delayed $\mathrm{D}_{n}$ networks}

We introduce a more general notation for delayed symmetrically coupled cell systems inspired by recent work on (non-necessarily symmetric) coupled cell systems of ordinary differential equations, see for instance [23]. Suppose that each cell in the system has phase space $\mathbb{R}^{k}$. We generalize systems (26) and (25) to

$$
\dot{X}_{i}(t)=f\left(\widetilde{X}_{i}, \widetilde{X}_{i+1}, \ldots, \widetilde{X}_{i+(n-1) / 2}, \widetilde{X}_{i-(n-1) / 2}, \ldots, \widetilde{X}_{i-1}\right), \quad i=1, \ldots, n
$$

and

$$
\dot{X}_{i}(t)=f\left(\widetilde{X}_{i}, \widetilde{X}_{i+1}, \ldots, \widetilde{X}_{i+(n / 2-1)}, \widetilde{X}_{i+n / 2}, \widetilde{X}_{i-(n / 2-1)}, \ldots, \widetilde{X}_{i-1}\right), \quad i=1, \ldots, n
$$

where

$$
\tilde{X}_{j}=\left(X_{j}\left(t-\tau_{1}\right), \ldots, X_{j}\left(t-\tau_{m}\right)\right),
$$

$f:\left(\mathbb{R}^{m}\right)^{n} \rightarrow \mathbb{R}^{k}$ is a smooth function and the position of $\widetilde{X}_{k}$ corresponds to the coupling from cell $k$ to cell $i$. We say that cells $j$ and $k$ have identical coupling to cell $i$ if

$$
f\left(X_{i}, \ldots, u, \ldots, v, \ldots\right)=f\left(X_{i}, \ldots, v, \ldots, u, \ldots\right)
$$

where $u$ and $v$ are permuted from positions $j$ and $k$. We rewrite systems (27) and (28) as

$$
\dot{X}=F(\widetilde{X})
$$

where $X=\left(X_{1}, \ldots, X_{n}\right)^{t}$

$$
\begin{aligned}
\widetilde{X} & =\widetilde{X}_{i}, \widetilde{X}_{i+1}, \ldots, \widetilde{X}_{i+(n-1) / 2}, \widetilde{X}_{i-(n-1) / 2}, \ldots, \widetilde{X}_{i-1}, \quad \text { or } \\
\widetilde{X} & =\widetilde{X}_{i}, \widetilde{X}_{i+1}, \ldots, \widetilde{X}_{i+(n / 2-1)}, \widetilde{X}_{i+n / 2}, \widetilde{X}_{i-(n / 2-1)}, \ldots, \widetilde{X}_{i-1},
\end{aligned}
$$

and $F:\left(\mathbb{R}^{m}\right)^{n} \rightarrow \mathbb{R}^{n k}$ has $i^{t h}$ component given by the formulas above for $\dot{X}_{i}(t)$ (for either $n$ odd or even). 
Consider the group $\mathbf{D}_{n}$, with generators $\rho$ and $\kappa$ acting on $\mathbb{R}^{k n}$ as follows:

$$
\begin{array}{ll}
\rho .\left(X_{1}, \ldots, X_{n}\right) & =\left(X_{n}, X_{1}, X_{2}, \ldots, X_{n-1}\right) \\
\kappa \cdot\left(X_{1}, \ldots, X_{n}\right)=\left(X_{1}, X_{n}, \ldots, X_{n+2-j}, \ldots, X_{(n+1) / 2}, X_{(n+3) / 2}, \ldots, X_{j}, \ldots, X_{2}\right) & \text { if } n \text { is odd } \\
\kappa \cdot\left(X_{1}, \ldots, X_{n}\right)=\left(X_{n}, \ldots, X_{n+1-j}, \ldots, X_{n / 2}, X_{n / 2+1}, \ldots, X_{j}, \ldots, X_{1}\right) & \text { if } n \text { is even. }
\end{array}
$$

These networks can be represented by a graph as shown in Figure 3.1.1. In this case, we have an eight-cell network with bidirectional nearest and next-nearest neighbour coupling with delays $\tau_{1}$ and $\tau_{2}$ respectively.

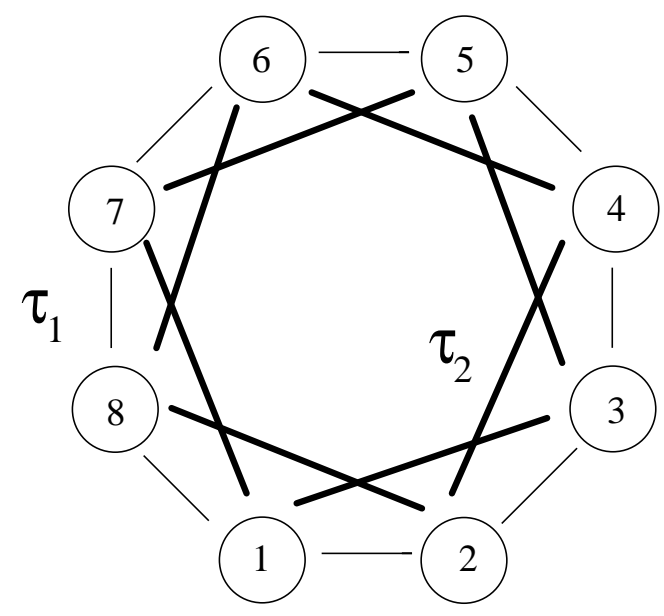

Figure 1: Representation of a $\mathbf{D}_{8}$-symmetric coupled cell system with nearest neighbour with delay $\tau_{1}$ and second nearest neighbour coupling with delay $\tau_{2}$.

Without loss of generality we assume that the networks are transitive. That is, all cells in the network can be reached from any other cell via the coupling arrows.

We now characterize the connections in the network so that the delay-differential system is $\mathbf{D}_{n}$-symmetric. We think of each cell in the network has having $[(n-1) / 2]$ neighbours on each side and an opposite cell if $n$ is even. Graphically, it is clear that a $n$-cell network is $\mathbf{D}_{n}$ symmetric if for all cells in the network, all connections to and from the $j^{\text {th }}$ neighbour on each side (or the opposite cell if $n$ is even) are all the same; that is, the coupling term and its delay must be the same for all those connections. This idea is formalized in the next result.

Proposition 3.3 A transitive network of $n$ coupled identical cells, with delays, is $\mathbf{D}_{n}$-equivariant if and only if it satisfies the conditions below.

(i) Suppose that cell 1 receives an input from cell $j$ with delay $\epsilon \in[0, \tau]$, then every cell $i$ in the network $(i=2, \ldots, n)$ receives an input from cell $(i+j-1) \bmod n$ with delay $\epsilon$ identical to the one received by cell 1.

(ii) For every connection in part (i), there is an identical connection from cell $i$ to cell $(i+j-$ 1) $\bmod n$ with delay $\epsilon$. 
From Proposition 3.3, a transitive $\mathbf{D}_{n}$ symmetric network with an even number of cells must have nearest neighbour coupling.

Proof: We begin by looking at $\rho$-equivariance. Denote by $[w]_{i}$ the $i^{\text {th }}$ row of vector $w$. Then,

$$
[\rho F(\widetilde{X})]_{i}=f\left(\widetilde{X}_{i-1}, \widetilde{X}_{i}, \ldots, \widetilde{X}_{i-2}\right) .
$$

and since $\rho X=\left(X_{n}, X_{1}, \ldots, X_{n-1}\right)$ we have

$$
[F(\rho \tilde{X})]_{i}=f\left(\widetilde{X}_{i-1}, \widetilde{X}_{i}, \ldots, \widetilde{X}_{i-2}\right) .
$$

Thus, $\rho$-equivariance holds automatically by the structure of the equations.

Consider the case $n$ odd (the case $n$ even has a similar proof and is omitted). Suppose that cell 1 receives an input from cell $j$. We look at the system of equation (27) and focus on the possible coupling from cell $(i+j-1) \bmod n$ to cell $i$. Moreover, consider the possible connection from cell $i$ to cell $(i-j+1) \bmod n$. Note that the connections from $(i+j-1) \bmod n$ to $i$ and from $i$ to $(i-j+1) \bmod n$ are obtained by taking the index and subtracting $j-1$. Finally, consider the possible connection from cell $(i-j+1) \bmod n$ to cell $i$. We now show that $F(\widetilde{X})$ is $\kappa$-equivariant (and so $\mathbf{D}_{n}$-equivariant) if and only if the connections defined above are identical. We see that

$$
\kappa F(\widetilde{X})=\kappa\left(\begin{array}{c}
f\left(\widetilde{X}_{1}, \ldots, \widetilde{X}_{j}, \ldots, \widetilde{X}_{n+2-j}, \ldots\right) \\
\vdots \\
f\left(\widetilde{X}_{i}, \ldots, \widetilde{X}_{i+j-1}, \ldots, \widetilde{X}_{i-j+1}, \ldots\right) \\
\vdots \\
f\left(\widetilde{X}_{n+2-i}, \ldots, \widetilde{X}_{n+2-i+j-1}, \ldots, \widetilde{X}_{n+2-i-j+1}, \ldots\right) \\
\vdots \\
f\left(\widetilde{X}_{1}, \ldots, \widetilde{X}_{j}, \ldots, \widetilde{X}_{n+2-j}, \ldots\right) \\
\vdots \\
\left.\tilde{X}_{n+2-i+j-1}, \ldots, \widetilde{X}_{n+2-i-j+1}, \ldots\right) \\
\vdots \\
f\left(\widetilde{X}_{i}, \ldots, \widetilde{X}_{i+j-1}, \ldots, \widetilde{X}_{i-j+1}, \ldots\right) \\
\vdots
\end{array}\right)
$$

and

$$
F(\kappa \widetilde{X})=\left(\begin{array}{c}
f\left(\widetilde{X}_{1}, \ldots, \widetilde{X}_{n+2-j}, \ldots, \widetilde{X}_{j}, \ldots\right) \\
\vdots \\
f\left(\widetilde{X}_{n+2-i}, \ldots, \widetilde{X}_{n+2-(i+j-1)}, \ldots, \widetilde{X}_{n+2-(i-j+1)}, \ldots\right) \\
\vdots \\
f\left(\widetilde{X}_{i}, \ldots, \widetilde{X}_{i-j+1}, \ldots, \widetilde{X}_{i+j-1}, \ldots\right) \\
\vdots
\end{array}\right)
$$


We now show that parts (i) and (ii) implies $\kappa$-equivariance. If part (i) holds, the coupling from cell $(i+j-1) \bmod n$ to cell $i$ and the coupling from cell $i$ to cell $(i-j+1) \bmod n$ are identical. Then, by part (ii), the coupling from cell $(i-j+1) \bmod n$ to cell $i$ is identical to the coupling from cell $i$ to cell $(i-j+1) \bmod n$. Therefore, the coupling from cells $(i+j-1) \bmod n$ and $(i-j+1) \bmod n$ to $i$ are identical. Since the dynamics of all cells is given by the same function $f$, this is true for all $i=1, \ldots, n$. Thus, $F$ is $\kappa$-equivariant.

Suppose now that $F$ is $\kappa$-equivariant. Equality of both sides of the equivariance condition implies that for all $i=1, \ldots, n$, the couplings from cells $(i+j-1) \bmod n$ and $(i-j+1) \bmod n$ to $i$ are identical. Since the dynamics of all cells is given by the same function $f$, the coupling from cell $(i+j-1) \bmod n$ to cell $i$ guarantees an identical coupling from cell $i$ to cell $(i-j+1) \bmod n$ and this proves (i). But, the coupling from cell $(i-j+1) \bmod n$ to $i$ is therefore identical to the coupling from cell $i$ to cell $(i-j+1) \bmod n$. Hence there is an identical two-way coupling between cells $i$ and $(i-j+1) \bmod n$ which proves (ii).

\subsubsection{General form of the characteristic equation}

We now focus our attention on delay-coupled cell systems where each cell is one-dimensional, that is $k=1$. We split the linear and nonlinear parts of systems (27, 28) and write the result in abstract form:

$$
\dot{X}=L X_{t}+H\left(X_{t}\right)
$$

where $X_{t} \in C\left([-\tau, 0], \mathbb{R}^{n}\right), L: C\left([-\tau, 0], \mathbb{R}^{n}\right) \rightarrow \mathbb{R}^{n}$ is a bounded linear map and $H$ is a nonlinear mapping. Thus, $L$ is $\mathbf{D}_{n}$-equivariant, $\eta(\theta)$ is a $n \times n \mathbf{D}_{n}$-equivariant matrix of bounded variation and

$$
L \phi=\int_{-\tau}^{0} d \eta(\theta) \phi
$$

Proposition 3.4 The matrix $\eta(\theta)$ is symmetric $\left(\eta(\theta)=\eta(\theta)^{T}\right)$ with the properties:

(1) for all $j=1, \ldots, n: \eta_{j j}(\theta)=p(\theta)$ for some function $p$,

(2) for all $i, k$ with $i \neq k$ then $\eta_{k i}(\theta)=\eta_{(2+n-k) i}(\theta)=\eta_{k 1}(\theta)$.

Proof: We use Proposition 3.3 to obtain information on $\eta$. By part (ii), the matrix $\eta(\theta)$ is symmetric. From the structure of (27/28), we deduce that for all $j=1, \ldots, n, \eta_{j j}(\theta)=p(\theta)$ for some function $p(\theta)$. We denote by $\eta_{j i}(\theta)$ the element of $\eta$ corresponding to the coupling from cell $j$ to $i$. Consider $\eta_{j 1}(\theta)$ then there is an identical connection from cell 1 to cell $2+n-j$ by part (i) and so $\eta_{j 1}(\theta)=\eta_{1(2+n-j)}(\theta)$. By part (ii), the connection from cell $2+n-j$ to cell 1 is identical to its reciprocal and so: $\eta_{j 1}(\theta)=\eta_{(2+n-j) 1}(\theta)$. By part (i), we then have $\eta_{k i}(\theta)=\eta_{(2+n-k) i}(\theta)=\eta_{k 1}(\theta)$ since the connections to cell $i$ are identical to the connections to cell 1 .

Remark 3.5 This result can be obtained for higher-dimensional cells with a proof essentially similar to this one, but with a more cumbersome notation. We decided to restrict ourselves to the one-dimensional case as this is the one which we study in details in what follows. 
For the purpose of diagonalizing the linear equation, it is preferable to use $\mathbb{C}^{n}$, the complexification of $\mathbb{R}^{n}$. The decomposition of $\mathbb{C}^{n}$ by the action of $\mathbf{D}_{n}=\langle\rho, \kappa\rangle$ yields an isotypic decomposition of $\mathbb{C}^{n}$, see Golubitsky et. al. [13]. Let $\zeta=e^{2 \pi i / n}$ and define

$$
V_{j}=\left\{\left[v, \zeta^{j} v, \zeta^{2 j} v, \ldots, \zeta^{(n-1) j} v\right]: v \in \mathbb{R}\right\}
$$

for $j=0, \ldots, n-1$. The subspaces $V_{j}$ are irreducible representations of $\mathbf{D}_{n}$. Recall that all complex irreducible representations of $\mathbf{D}_{n}$ are one-dimensional. Then the isotypic decomposition is

$$
\mathbb{C}^{n}=V_{0} \oplus V_{1} \oplus \ldots \oplus V_{n-1}
$$

where $V_{0}$ is the trivial representation and for $n$ even $V_{n / 2}$ is the alternating representation both of which are real one-dimensional irreducible representations of $\mathbf{D}_{n}$.

Using Proposition 3.4 and by invariance of the subspace $V_{j}$ we have

$$
\eta(\theta)\left[v, \zeta^{j} v, \zeta^{2 j} v, \ldots, \zeta^{(n-1) j} v\right]=\left(p(\theta)+\sum_{k=2}^{n}\left(\zeta^{(k-1) j}+\zeta^{(n+1-k) j}\right) \eta_{k 1}(\theta)\right)\left[v, \zeta^{j} v, \zeta^{2 j} v, \ldots, \zeta^{(n-1) j} v\right] .
$$

Since $\zeta^{n-(k-1)}=\overline{\zeta^{k-1}}$, then for $j=0, \ldots, n-1$

$$
\begin{aligned}
A_{j}(\theta) & :=p(\theta)+\sum_{k=2}^{n}\left(\zeta^{(k-1) j}+\zeta^{(n+1-k) j}\right) \eta_{k 1}(\theta) \\
& =p(\theta)+\sum_{k=2}^{n} 2 \cos (2 \pi(k-1) j / n) \eta_{k 1}(\theta) \\
& = \begin{cases}p(\theta)+\sum_{k=2}^{(n+1) / 2} 4 \cos (2 \pi(k-1) j / n) \eta_{k 1}(\theta) & n \text { odd } \\
p(\theta)+2(-1)^{j} \eta_{(1+n / 2) 1}(\theta)+\sum_{k=2}^{n / 2} 4 \cos (2 \pi(k-1) j / n) \eta_{k 1}(\theta) & n \text { even }\end{cases}
\end{aligned}
$$

since $\eta_{k 1}=\eta_{(2+n-k) 1}$ and $\cos (2 \pi(k-1) j / n)=\cos (2 \pi((2+n-k)-1) j / n)$. Note that $A_{j}(\theta)=$ $A_{n-j}(\theta)$ for $j=1, \ldots,[n / 2]$. The block diagonalization of $\eta$ is given by the terms $A_{j}(\theta)$ for $j=0, \ldots, n-1$. Hence, we have

$$
\Delta(\lambda)=\lambda I_{n}-\int_{-\tau}^{0} d \eta(\theta) e^{\lambda \theta}=\lambda I_{n}-\int_{-\tau}^{0} \operatorname{diag}\left(d A_{0}(\theta) e^{\lambda \theta}, \ldots, d A_{n-1}(\theta) e^{\lambda \theta}\right) .
$$

Let $\Delta_{j}(\lambda)=\lambda-\int_{-\tau}^{0} d A_{j}(\theta) e^{\lambda \theta}$, then

$$
\Delta(\lambda)=\operatorname{diag}\left(\Delta_{0}(\lambda), \ldots, \Delta_{n-1}(\lambda)\right) .
$$

Therefore, the characteristic equation has, for $n$ even, the decomposition

$$
\operatorname{det} \Delta(\lambda)=\operatorname{det} \Delta_{0}(\lambda) \operatorname{det} \Delta_{n / 2}(\lambda) \prod_{j=1}^{(n-1) / 2}\left[\operatorname{det} \Delta_{j}(\lambda)\right]^{2}=0
$$


and for $n$ odd

$$
\operatorname{det} \Delta(\lambda)=\operatorname{det} \Delta_{0}(\lambda) \prod_{j=1}^{(n-1) / 2}\left[\operatorname{det} \Delta_{j}(\lambda)\right]^{2}=0
$$

\subsubsection{Application of Main Theorems to the $\mathbf{D}_{n}$ case}

It is straightforward that Theorem 2.1 can be applied to any of the factors of the characteristic equations (32) and (33).

To apply Theorem 2.4 in the $\mathbf{D}_{n}$ case, we need to verify that the coefficients $b_{k}^{j}$ in the factors of the characteristic equation are nonzero and that the nondegeneracy condition $\operatorname{det} \mathcal{I}_{B} \neq 0$ is satisfied. In fact, as it is shown in Section $4, \operatorname{det} \mathcal{I}_{B} \neq 0$ if and only if the matrix

$$
\mathcal{B}:=\left[\begin{array}{cccc}
b_{1}^{1} & b_{1+\mu_{1}}^{1} & \cdots & b_{1+\mu_{r-1}}^{1} \\
b_{1}^{2} & b_{1+\mu_{1}}^{2} & \cdots & b_{1+\mu_{r-1}}^{2} \\
\vdots & \vdots & \ddots & \vdots \\
b_{1}^{r} & b_{1+\mu_{1}}^{r} & \cdots & b_{1+\mu_{r-1}}^{r}
\end{array}\right] .
$$

is nonsingular where $\ell_{j}$ is the number of imaginary eigenvalues satisfying the $j^{\text {th }}$ term of the product of the characteristic equation (21) and $\mu_{j}=\sum_{i=1}^{j} \ell_{i}$ where $\mu_{r}=n$ and $\mu_{0}:=0$. Note that row $j$ of $\mathcal{B}$ contains coefficients belonging to the $j^{\text {th }}$ factor of the characteristic equation (21). The cases $n$ even and $n$ odd differ significantly and we focus initially on the case $n$ odd.

\subsection{Critical eigenvalues of $\mathrm{D}_{n}$ coupled cell systems}

In this section, we apply Theorem 2.4 to $\mathbf{D}_{n}$-symmetric coupled cell system depending on an arbitrary number of finite delays.

\subsection{1 $n$ odd}

The characteristic equation is

$$
\operatorname{det} \Delta(\lambda)=\operatorname{det} \Delta_{0}(\lambda) \prod_{j=1}^{(n-1) / 2}\left[\operatorname{det} \Delta_{j}(\lambda)\right]^{2}=0 .
$$

We can write

$$
\Delta_{j}(\lambda)=\lambda-F(\lambda)-G_{j}(\lambda)
$$

where

$$
F(\lambda)=\sum_{i=1}^{p} a_{i} e^{-\lambda \tau_{i}}
$$

are the terms coming from the internal dynamics of each cell and

$$
G_{j}(\lambda)=\sum_{k=2}^{(n+1) / 2}\left[4 \cos \left(\frac{2 \pi(k-1) j}{n}\right)\right] \sum_{t=1}^{m_{k}} \alpha_{t}^{k} e^{-\lambda s_{t}^{k}} .
$$


are the contributions from the coupling where $m_{k}$ is the number of delayed terms in the connection from cell $k$ to 1 and $\alpha_{t}^{k}$ are the respective coupling coefficients.

Example 3.6 As an example, consider a delay-coupled $\mathbf{D}_{5}$-symmetric cell. Let $u_{s}(\theta)=0$ if $\theta=[-\tau,-s]$ and $u_{s}(\theta)=1$ for $\theta \in(-s, 0]$ where $\tau \geq s$ for all delays $s$ and suppose

$$
\eta(\theta)=\left[\begin{array}{ccccc}
p(\theta) & \eta_{21}(\theta) & \eta_{31}(\theta) & \eta_{41}(\theta) & \eta_{51}(\theta) \\
\eta_{21}(\theta) & p(\theta) & \eta_{51}(\theta) & \eta_{31}(\theta) & \eta_{41}(\theta) \\
\eta_{41}(\theta) & \eta_{21}(\theta) & p(\theta) & \eta_{51}(\theta) & \eta_{31}(\theta) \\
\eta_{31}(\theta) & \eta_{41}(\theta) & \eta_{21}(\theta) & p(\theta) & \eta_{51}(\theta) \\
\eta_{51}(\theta) & \eta_{41}(\theta) & \eta_{31}(\theta) & \eta_{21}(\theta) & p(\theta)
\end{array}\right]
$$

where

$$
p(\theta)=\sum_{i=1}^{2} a_{i} u_{\tau_{i}}(\theta), \quad \eta_{21}(\theta)=\sum_{\ell=1}^{3} \alpha_{\ell}^{2} u_{s_{\ell}^{2}}(\theta) \quad \text { and } \quad \eta_{31}(\theta)=\sum_{\ell=1}^{2} \alpha_{\ell}^{3} u_{s_{\ell}^{3}}(\theta)
$$

with the conditions $\eta_{41}(\theta)=\eta_{31}(\theta)$ and $\eta_{51}(\theta)=\eta_{21}(\theta)$ given by Proposition 3.4 part (2). Then,

$$
F(\lambda)=\sum_{i=1}^{2} a_{i} e^{-\lambda \tau_{i}}
$$

and

$$
G_{j}(\lambda)=\sum_{k=2}^{3}\left[4 \cos \left(\frac{2 \pi(k-1) j}{n}\right)\right] \sum_{t=1}^{m_{k}} \alpha_{t}^{k} e^{-\lambda s_{t}^{k}}
$$

where $m_{2}=3$ and $m_{3}=2$.

Thus, all coefficients $b_{j}^{k}$ of $\mathcal{I}_{B}$ are nonzero and it is convenient to set $b_{1}^{j}$ to be the coefficient of $a_{1}$; that is $b_{1}^{j}=1$ for $j=0,1,2, \ldots,(n+1) / 2$, and we keep this convention for the remainder of the paper.

We suppose that the characteristic equation $\Delta(\lambda)=0$ has purely imaginary roots coming from all factors, then for $r=(n-1) / 2$ we have

$$
\mathcal{B}:=\left[\begin{array}{cccc}
b_{1}^{1} & b_{1+\mu_{1}}^{1} & \cdots & b_{1+\mu_{r-1}}^{1} \\
b_{1}^{2} & b_{1+\mu_{1}}^{2} & \cdots & b_{1+\mu_{r-1}}^{2} \\
\vdots & \vdots & \ddots & \vdots \\
b_{1}^{r} & b_{1+\mu_{1}}^{r} & \cdots & b_{1+\mu_{r-1}}^{r}
\end{array}\right] .
$$

and we assign the coefficients $b_{1+\mu_{j}}^{i}$ as follows. We suppose that the first row corresponds to the factor for the trivial representation which means that

$$
b_{1+\mu_{j}}^{1}=4, \quad j=1,2, \ldots,(n-1) / 2 .
$$


Then, we set the remaining coefficients of each row to be equal to $\left[4 \cos \left(\frac{2 \pi(k-1) j}{n}\right)\right]$ for $k=2,3, \ldots,(n+1) / 2$ where row $j+1$ has the coefficients of $\Delta_{j}$ for $j=1,2, \ldots,(n-1) / 2$. This leads to the matrix

$$
\mathcal{B}=\left[\begin{array}{cccccc}
1 & 4 & 4 & \cdots & 4 & 4 \\
1 & 4 \cos \left(\frac{2 \pi}{n}\right) & 4 \cos \left(\frac{4 \pi}{n}\right) & \cdots & 4 \cos \left(\frac{(n-3) \pi}{n}\right) & 4 \cos \left(\frac{(n-1) \pi}{n}\right) \\
1 & 4 \cos \left(\frac{4 \pi}{n}\right) & 4 \cos \left(\frac{8 \pi}{n}\right) & \cdots & 4 \cos \left(\frac{2(n-3) \pi}{n}\right) & 4 \cos \left(\frac{2(n-1) \pi}{n}\right) \\
\vdots & \vdots & \vdots & \vdots & \vdots \\
1 & 4 \cos \left(\frac{(n-1) \pi}{n}\right) & 4 \cos \left(\frac{2(n-1) \pi}{n}\right) & \cdots & 4 \cos \left(\frac{(n-3)(n-1) \pi}{2 n}\right) & 4 \cos \left(\frac{(n-1)^{2} \pi}{2 n}\right)
\end{array}\right] .
$$

Let $i_{1}<i_{2}<\ldots<i_{s}$ be a set of indices chosen from $\{0, \ldots,(n-1) / 2\}$ defining a combination of factors from the characteristic equation (33). We now construct the $s \times s$ matrix $\mathcal{B}$ by removing rows and columns of (35) not in the set $\left\{i_{1}, i_{2}, \ldots, i_{s}\right\}$. Suppose that $i_{1}, \ldots, i_{s}$ are chosen from $\{1, \ldots,(n-1) / 2\}$ then the matrix $\mathcal{B}$ is symmetric $\left(\mathcal{B}^{T}=\mathcal{B}\right)$ and has the form

$$
\mathcal{B}=\left[\begin{array}{ccccc}
4 \cos \left(\frac{2 \pi i_{1}^{2}}{n}\right) & 4 \cos \left(\frac{2 \pi i_{2} i_{1}}{n}\right) & \cdots & 4 \cos \left(\frac{2 \pi i_{s-1} i_{1}}{n}\right) & 4 \cos \left(\frac{2 \pi i_{s} i_{1}}{n}\right) \\
4 \cos \left(\frac{2 \pi i_{1} i_{2}}{n}\right) & 4 \cos \left(\frac{2 \pi i_{2}^{2}}{n}\right) & \cdots & 4 \cos \left(\frac{2 \pi i_{s-1} i_{2}}{n}\right) & 4 \cos \left(\frac{2 \pi i_{s} i_{2}}{n}\right) \\
\vdots & \vdots & \vdots & \vdots & \vdots \\
4 \cos \left(\frac{2 \pi i_{1} i_{s-1}}{n}\right) & 4 \cos \left(\frac{2 \pi i_{2} i_{s-1}}{n}\right) & \cdots & 4 \cos \left(\frac{2 \pi i_{s-1}^{2}}{n}\right) & 4 \cos \left(\frac{2 \pi i_{s} i_{s-1}}{n}\right) \\
4 \cos \left(\frac{2 \pi i_{1} i_{s}}{n}\right) & 4 \cos \left(\frac{2 \pi i_{2} i_{s}}{n}\right) & \cdots & 4 \cos \left(\frac{2 \pi i_{s-1} i_{s}}{n}\right) & 4 \cos \left(\frac{2 \pi i_{s}^{2}}{n}\right) .
\end{array}\right] .
$$

In the other case, $i_{1}=0$ and the matrix is of the form 


$$
\mathcal{B}=\left[\begin{array}{ccccc}
1 & 4 & \cdots & 4 & 4 \\
1 & 4 \cos \left(\frac{2 \pi i_{2}^{2}}{n}\right) & \cdots & 4 \cos \left(\frac{2 \pi i_{s-1} i_{2}-1}{n}\right) & 4 \cos \left(\frac{2 \pi i_{s} i_{2}}{n}\right) \\
\vdots & \vdots & \vdots & \vdots & \vdots \\
1 & 4 \cos \left(\frac{2 \pi i_{2} i_{s-1}}{n}\right) & \cdots & 4 \cos \left(\frac{2 \pi i_{s-1} i_{s-1}}{n}\right) & 4 \cos \left(\frac{2 \pi i_{s} i_{s-1}}{n}\right) \\
1 & 4 \cos \left(\frac{2 \pi i_{2} i_{s}}{n}\right) & \cdots & 4 \cos \left(\frac{2 \pi i_{s-1}^{2}}{n}\right) & 4 \cos \left(\frac{2 \pi i_{s}^{2}}{n}\right) .
\end{array}\right]
$$

We can now state our result.

Theorem 3.7 Consider a linear $\mathbf{D}_{n}$-symmetric coupled cell system with $n$ odd depending on $k$ delays $\tau_{1}, \ldots, \tau_{k}$ and let $i_{1}<i_{2}<\ldots<i_{s}$ be indices chosen from $\{0, \ldots,(n-1) / 2\}$ defining a combination of factors from the characteristic equation (33). We assume that the matrix $\mathcal{B}$ given by (36) or (37) is nonsingular. Suppose

$$
\omega_{1}^{1}, \ldots, \omega_{\ell_{i_{1}}}^{1}, \omega_{1}^{2}, \ldots, \omega_{\ell_{i_{2}}}^{2} \ldots, \omega_{1}^{s}, \ldots, \omega_{\ell_{i_{s}}}^{s}
$$

are positive and linearly independent over the rationals, where $\ell_{i_{1}}+\cdots+\ell_{i_{s}}=k$. Then there exists $\tau_{1}>0, \ldots, \tau_{k}>0$ and real coefficients $a_{i}$ such that for all $m=1, \ldots, s$

$$
\Delta_{i_{m}}(\lambda)=0
$$

has roots $i \omega_{\ell}^{m}$ for $\ell=1, \ldots, \ell_{i_{m}}$.

Proof: $\quad$ Since $n$ is odd, the coefficients

$$
b_{k}^{j}=4 \cos \left(\frac{2 \pi(k-1) j}{n}\right)
$$

are nonzero for all $k=2, \ldots,(n+1) / 2$ and $j=0, \ldots,(n-1) / 2$. Because $\mathcal{B}$ is assumed nonsingular, Theorem 2.4 applies and the result is obtained.

The condition that $\mathcal{B}$ is nonsingular does not always hold as we show in the case $s=2$. Consider the matrix (37) with $n=9$ so that $i_{2} \in\{1,2,3,4\}$. Choosing $i_{2}=3$ we have the singular matrix

$$
\mathcal{B}=\left(\begin{array}{ll}
1 & 4 \\
1 & 4
\end{array}\right) \text {. }
$$


We now show that $\mathcal{B}$ is nonsingular in the case $s=2$ if the matrix is given by (36); that is,

$$
\mathcal{B}=\left(\begin{array}{cc}
4 \cos \left(\frac{2 \pi i_{1}^{2}}{n}\right) & 4 \cos \left(\frac{2 \pi i_{1} i_{2}}{n}\right) \\
4 \cos \left(\frac{2 \pi i_{2} i_{1}}{n}\right) & 4 \cos \left(\frac{2 \pi i_{2}^{2}}{n}\right)
\end{array}\right) .
$$

We compute

$$
\begin{aligned}
\operatorname{det} \mathcal{B} & =16\left[\cos \left(\frac{2 \pi i_{1}^{2}}{n}\right) \cos \left(\frac{2 \pi i_{2}^{2}}{n}\right)-\cos \left(\frac{2 \pi i_{1} i_{2}}{n}\right)^{2}\right] \\
& =8\left[\cos \left(\frac{2 \pi\left(i_{1}^{2}+i_{2}^{2}\right)}{n}\right)+\cos \left(\frac{2 \pi\left(i_{1}^{2}-i_{2}^{2}\right)}{n}\right)-\cos \left(\frac{4 \pi i_{1} i_{2}}{n}\right)-1\right] .
\end{aligned}
$$

We do a few cases explicitly. First, the case $n=3$ is not relevant since $i_{1}<i_{2},(n-1) / 2=1$ and $i_{1} \neq 0$. We do the case $n=5$ where we must have $i_{1}=1$ and $i_{2}=2$. This implies that $i_{1}^{2}+i_{2}^{2}=5$ and so

$$
\operatorname{det} \mathcal{B}=8\left[\cos \left(\frac{4 \pi}{5}\right)-\cos \left(\frac{8 \pi}{5}\right)\right] \neq 0
$$

We now turn to the general case and show that the determinant cannot vanish. Because the three cosines are projections of $n^{\text {th }}$ roots of unity on the real axis for $n$ odd, then

$$
\cos \left(\frac{2 \pi\left(i_{1}^{2}+i_{2}^{2}\right)}{n}\right)+\cos \left(\frac{2 \pi\left(i_{1}^{2}-i_{2}^{2}\right)}{n}\right)-\cos \left(\frac{4 \pi i_{1} i_{2}}{n}\right) \neq 1
$$

So if the determinant is to vanish, one of the cosines must be equal to 1 . Since $i_{1}<i_{2}$ there is only one option and we must have $i_{1}^{2}+i_{2}^{2}=n$. Thus, $i_{1}^{2}=n-i_{2}^{2}$ and

$$
\cos \left(\frac{2 \pi\left(i_{1}^{2}-i_{2}^{2}\right)}{n}\right)=\cos \left(\frac{2 \pi\left(n-2 i_{2}^{2}\right)}{n}\right)=\cos \left(\frac{4 \pi i_{2}^{2}}{n}\right) .
$$

If

$$
\cos \left(\frac{4 \pi i_{2}^{2}}{n}\right)-\cos \left(\frac{4 \pi i_{1} i_{2}}{n}\right)=0,
$$

this would impliy $i_{1}=i_{2}$, but we know that $i_{1}<i_{2}$ and so $\operatorname{det} \mathcal{B}$ cannot vanish. We summarize this result in the next theorem.

Theorem 3.8 Consider a linear $\mathbf{D}_{n}$-symmetric coupled cell system with $n$ odd depending on $k$ delays $\tau_{1}, \ldots, \tau_{k}$ and let $i_{1}<i_{2}$ be indices chosen from $\{1, \ldots,(n-1) / 2\}$ defining a combination of factors from the characteristic equation (33). Suppose

$$
\omega_{1}^{1}, \ldots, \omega_{\ell_{i_{1}}}^{1}, \omega_{1}^{2}, \ldots, \omega_{\ell_{i_{2}}}^{2}
$$


are positive and linearly independent over the rationals, where $\ell_{i_{1}}+\ell_{i_{2}}=k$. Then there exists $\tau_{1}>0, \ldots, \tau_{k}>0$ and real coefficients $a_{1} \in \mathbb{R}, \ldots, a_{p} \in \mathbb{R}$ such that for $m=1$ and $m=2$,

$$
\Delta_{i_{m}}(\lambda)=0
$$

has roots $i \omega_{\ell}^{m}$ for $\ell=1, \ldots, \ell_{i_{m}}$.

\section{$3.3 n$ even}

For $\mathbf{D}_{n}$-symmetric systems with $n$ even, Theorem 2.4 cannot be applied directly because the condition $b_{k}^{j} \neq 0$ for all indices $j, k$ is not always satisfied. For instance, if $n=4$ then

$$
A_{j}(\theta)=p(\theta)+2(-1)^{j} \eta_{31}(\theta)+4 \cos (2 \pi j / 4) \eta_{21}(\theta)
$$

and we have

$$
A_{1}(\theta)=A_{3}(\theta)=p(\theta)-2 \eta_{31}(\theta) .
$$

Hence, $b_{2}^{1}=b_{2}^{3}=0$.

\section{Proof of Theorem 2.4}

Before we present the proof of Theorem 2.4, we describe in the next lemma the form of the matrix $\mathcal{I}_{B}$ which appears in the proof and compute its determinant.

Lemma 4.1 Let $\ell_{1}, \ldots, \ell_{r}$ be positive integers and define $\mu_{j}=\sum_{i=1}^{j} \ell_{i}$ where $\mu_{r}=n$ and $\mu_{0}:=0$. Consider the $n \times n$ matrix

$$
\mathcal{I}_{B}:=\left[A_{1} \cdots A_{j} \cdots A_{r}\right]^{T}
$$

where

$$
A_{j}=\left[\begin{array}{cccccccccc}
b_{1}^{j} & \cdots & b_{\mu_{j-1}}^{j} & b_{1+\mu_{j-1}}^{j} & b_{2+\mu_{j-1}}^{j} & \cdots & b_{\mu_{j}}^{j} & b_{\mu_{j}+1}^{j} & \cdots & b_{n}^{j} \\
b_{1}^{j} & \cdots & b_{\mu_{j-1}}^{j} & b_{1+\mu_{j-1}}^{j} & b_{2+\mu_{j-1}}^{j} & \cdots & -b_{\mu_{j}}^{j} & b_{\mu_{j}+1}^{j} & \cdots & b_{n}^{j} \\
\vdots & \cdots & \vdots & \vdots & \vdots & \cdots & \vdots & \vdots & \cdots & \vdots \\
b_{1}^{j} & \cdots & b_{\mu_{j-1}}^{j} & b_{1+\mu_{j-1}}^{j} & -b_{2+\mu_{j-1}}^{j} & \cdots & -b_{\mu_{j}}^{j} & b_{\mu_{j}+1}^{j} & \cdots & b_{n}^{j}
\end{array}\right]
$$

is a $\ell_{j} \times n$ matrix and all elements are nonzero. Then,

$$
\operatorname{det} \mathcal{I}_{B}= \pm \prod_{j=1}^{r}\left[(-2)^{\ell_{j}-1} \prod_{s=2}^{\ell_{j}} b_{s+\mu_{j-1}}^{j}\right] \operatorname{det} \mathcal{B}
$$

where

$$
\mathcal{B}:=\left[\begin{array}{cccc}
b_{1}^{1} & b_{1+\mu_{1}}^{1} & \cdots & b_{1+\mu_{r-1}}^{1} \\
b_{1}^{2} & b_{1+\mu_{1}}^{2} & \cdots & b_{1+\mu_{r-1}}^{2} \\
\vdots & \vdots & \ddots & \vdots \\
b_{1}^{r} & b_{1+\mu_{1}}^{r} & \cdots & b_{1+\mu_{r-1}}^{r}
\end{array}\right]
$$


Proof: $\quad$ Substitute row $k$, denoted by $R_{k}$, of

$$
A_{j}=\left[\begin{array}{cccccccccc}
b_{1}^{j} & \cdots & b_{\mu_{j-1}}^{j} & b_{1+\mu_{j-1}}^{j} & b_{2+\mu_{j-1}}^{j} & \cdots & b_{\mu_{j}}^{j} & b_{\mu_{j}+1}^{j} & \cdots & b_{n}^{j} \\
b_{1}^{j} & \cdots & b_{\mu_{j-1}}^{j} & b_{1+\mu_{j-1}}^{j} & b_{2+\mu_{j-1}}^{j} & \cdots & -b_{\mu_{j}}^{j} & b_{\mu_{j}+1}^{j} & \cdots & b_{n}^{j} \\
\vdots & \cdots & \vdots & \vdots & \vdots & \cdots & \vdots & \vdots & \cdots & \vdots \\
b_{1}^{j} & \cdots & b_{\mu_{j-1}}^{j} & b_{1+\mu_{j-1}}^{j} & -b_{2+\mu_{j-1}}^{j} & \cdots & -b_{\mu_{j}}^{j} & b_{\mu_{j}+1}^{j} & \cdots & b_{n}^{j}
\end{array}\right]
$$

for $k=2, \ldots, \ell_{j}$ by $R_{k}-R_{1}$. The matrix $A_{j}$ becomes

$$
\tilde{A}_{j}:=\left[\begin{array}{cccccccccccc}
b_{1}^{j} & \cdots & b_{\mu_{j-1}}^{j} & b_{1+\mu_{j-1}}^{j} & b_{2+\mu_{j-1}}^{j} & b_{3+\mu_{j-1}}^{j} & \cdots & b_{-1+\mu_{j}}^{j} & b_{\mu_{j}}^{j} & b_{\mu_{j}+1}^{j} & \cdots & b_{n}^{j} \\
0 & \cdots & 0 & 0 & 0 & 0 & \cdots & 0 & -2 b_{\mu_{j}}^{j} & 0 & \cdots & 0 \\
0 & \cdots & 0 & 0 & 0 & 0 & \cdots & -2 b_{-1+\mu_{j}}^{j} & -2 b_{\mu_{j}}^{j} & 0 & \cdots & 0 \\
\vdots & \cdots & \vdots & \vdots & \vdots & \vdots & \cdots & \vdots & \vdots & \vdots & \cdots & \vdots \\
0 & \cdots & 0 & 0 & 0 & -2 b_{3+\mu_{j-1}}^{j} & \cdots & -2 b_{-1+\mu_{j}}^{j} & -2 b_{\mu_{j}}^{j} & 0 & \cdots & 0 \\
0 & \cdots & 0 & 0 & -2 b_{2+\mu_{j-1}}^{j} & -2 b_{3+\mu_{j-1}}^{j} & \cdots & -2 b_{-1+\mu_{j}}^{j} & -2 b_{\mu_{j}}^{j} & 0 & \cdots & 0
\end{array}\right]
$$

We compute the determinant of $\mathcal{I}_{B}$ by cofactor expansion starting with row 2 of $\tilde{A}_{j}$ which contains a unique nonzero element $-2 b_{\mu_{j}}^{j}$. Denote by $C_{i j}$ the $(i, j)$-cofactor matrix. The row $2+\mu_{j-1}$ of $C_{\left(2+\ell_{j-1}, \mu_{j}\right)}$ has a unique nonzero element $-2 b_{-1+\mu_{j}}$ and we perform a cofactor expansion along this row. The row $2+\mu_{j-1}$ of this new cofactor matrix also has a unique nonzero element $-2 b_{-2+\mu_{j}}^{j}$ and we proceed with the same process removing successively columns $3+\mu_{j-1}$ to $\mu_{j}$ (and the appropriate rows) until the cofactor matrix has only two rows corresponding to the original $\tilde{A}_{j}$ matrix and the second row has the unique nonzero element $-2 b_{2+\mu_{j-1}}$ which is used to perform a cofactor expansion. Performing this process successively on each matrix $\tilde{A}_{j}$ for $j=1, \ldots, r$, leaves as a cofactor matrix the $r \times r$ matrix $\mathcal{B}$ defined in the statement. The formula in the lemma is written using $\mu_{j}=\mu_{j-1}+\ell_{j}$ and so the lemma is proved.

We are now ready to prove our Theorem 2.4.

Proof of Theorem 2.4 A necessary and sufficient condition for the conclusion of the theorem to hold is that the following algebraic system of $2 n$ equations has a solution in the $2 n$ unknowns $\left(\tau_{1}, \tau_{2}, \ldots, \tau_{n}, a_{1}, a_{2}, \ldots, a_{n}\right)$ :

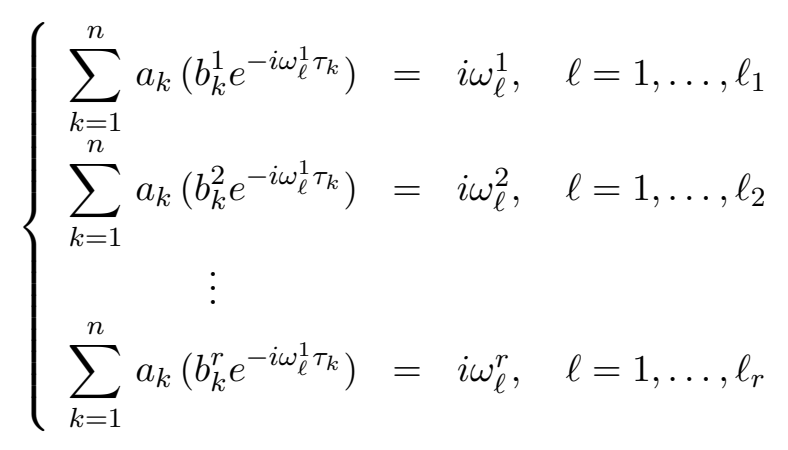




$$
\left\{\begin{array}{ccc}
\sum_{k=1}^{n} a_{k}\left(b_{k}^{1} e^{i \omega_{\ell}^{1} \tau_{k}}\right) & =-i \omega_{\ell}^{1}, \quad k=1, \ldots, \ell_{1} \\
\sum_{k=1}^{n} a_{k}\left(b_{k}^{2} e^{i \omega_{\ell}^{1} \tau_{k}}\right) & =-i \omega_{\ell}^{2}, \quad \ell=1, \ldots, \ell_{2} \\
\vdots & \\
\sum_{k=1}^{n} a_{k}\left(b_{k}^{r} e^{i \omega_{\ell}^{1} \tau_{k}}\right) & =-i \omega_{\ell}^{r}, \quad \ell=1, \ldots, \ell_{r}
\end{array}\right.
$$

Although (39) is in complex form, system (39) is equivalent to a system of $2 n$ real equations. This fact is taken for granted throughout the sequel, even though we continue to use complex notation.

Let

$$
\omega=\left(\omega_{1}^{1}, \ldots, \omega_{\ell_{1}}^{1}, \ldots, \omega_{1}^{r}, \ldots, \omega_{\ell_{r}}^{r}\right) .
$$

It is useful to use the following matrix notation for (39)

$$
\left(\begin{array}{c}
P(\tau ; \omega) \\
P(-\tau ; \omega)
\end{array}\right) A^{T}=\left(\begin{array}{c}
i \omega^{T} \\
-i \omega^{T}
\end{array}\right)
$$

where $A=\left(a_{1}, \ldots, a_{n}\right)$, superscript $T$ denotes transpose, and $P(\tau ; \omega)=P\left(\tau_{1}, \ldots, \tau_{n} ; \omega\right)$ is the $n \times n$ matrix of the form

$$
P(\tau ; \omega)=\left[\begin{array}{c}
P_{1}(\tau ; \omega) \\
P_{2}(\tau ; \omega) \\
\vdots \\
P_{r}(\tau ; \omega)
\end{array}\right]
$$

whose entry at block $j$, row $\ell$ and column $k$ is

$$
\left[P_{j}(\tau ; \omega)\right]_{\ell k}=b_{k}^{j} e^{-i \omega_{\ell}^{j} \tau_{k}} .
$$

Note that $\overline{P(\tau ; \omega)}=P(-\tau ; \omega)$.

Recall that $\mathrm{T}=\left(\mathbb{S}^{1}\right)^{n}$ and $\mathrm{V}=\mathrm{T}^{n}$. Consider the following mapping associated to (40):

$$
F: \mathrm{V} \times \mathbb{R}^{n} \longmapsto \mathbb{R}^{2 n}
$$

defined as follows.

$$
F\left(\Phi^{1}, \ldots, \Phi^{r}, A ; \omega\right)=\left(\begin{array}{c}
\widetilde{P}\left(\Phi^{1}, \ldots, \Phi^{r}\right) \\
\widetilde{P}\left(-\Phi^{1}, \ldots,-\Phi^{r}\right)
\end{array}\right) A^{T}-i\left(\begin{array}{c}
\omega^{T} \\
-\omega^{T}
\end{array}\right),
$$

where $A$ and $\omega$ are as previously defined and

$$
\widetilde{P}\left(\Phi^{1}, \ldots, \Phi^{r}\right)=\left[\begin{array}{c}
\widetilde{P}_{1}\left(\Phi^{1}\right) \\
\widetilde{P}_{2}\left(\Phi^{2}\right) \\
\vdots \\
\widetilde{P}_{r}\left(\Phi^{r}\right)
\end{array}\right]
$$


with

$$
\left[\widetilde{P}_{j}\left(\Phi^{1}, \ldots, \Phi^{n}\right)\right]_{\ell k}=b_{k}^{j} e^{-i \varphi_{\ell k}^{j}}
$$

for $j=1, \ldots, r, \ell=1, \ldots, \ell_{j}$ and $k=1, \ldots, n$. The definition of $\widetilde{P}$ uses the following coordinates of $\mathrm{V}$. Let $\mathrm{T}_{j}=\left(\mathbb{S}^{1}\right)^{\ell_{j}}$ for $j=1, \ldots, r$, then

$$
\left(\Phi^{1}, \ldots, \Phi^{r}\right) \in \mathrm{T}_{j}^{n} \times \cdots \times \mathrm{T}_{j}^{n}
$$

where

$$
\Phi^{j}=\left(\Phi_{1}^{j}, \ldots, \Phi_{n}^{j}\right) \quad \text { and } \quad \Phi_{k}^{j}=\left(\varphi_{1 k}^{j}, \ldots, \varphi_{\ell_{j}}^{j}\right)^{T} \in \mathrm{T}_{j} .
$$

Let $\Psi_{j}=\Phi_{n}^{j}$ and $\Psi=\left(\Psi_{1}, \ldots, \Psi_{r}\right)$. We use the notation

$$
\Phi=\left(\Phi_{o}^{1}, \ldots, \Phi_{o}^{n-1}\right)
$$

where

$$
\Phi_{o}^{j}=\left(\Phi_{1}^{j}, \ldots, \Phi_{n-1}^{j}\right)
$$

so that the mapping $F$ in (41) is written as $F(\Phi, \Psi, A ; \omega)$.

Definition: If $\left\{e_{1}, \ldots, e_{\ell}\right\}$ denotes the canonical basis of vectors in $\mathbb{R}^{\ell}$, we define the vectors $v_{1}, \ldots, v_{\ell}$ by $v_{1}=e_{1}+\cdots+e_{\ell}$, and for $k=2, \ldots, \ell$,

$$
v_{k}=v_{1}-\sum_{m=0}^{\ell-2} 2 e_{\ell-m} .
$$

By construction, the set $\left\{v_{1}, \ldots, v_{\ell}\right\}$ is linearly independent and so the $\ell \times \ell$ matrix $\mathcal{I}^{\ell}$, whose $j^{\text {th }}$ column is the vector $v_{j}^{T}$, is invertible.

We also define the $\ell \times \ell$ invertible matrix $\mathcal{U}_{j}$ to be the diagonal matrix whose $k^{\text {th }}$ diagonal element is the $k^{\text {th }}$ component of the vector $v_{j}$ (in particular, $\mathcal{U}_{1}$ is the identity matrix). Note also that $\mathcal{U}_{j}^{-1}=\mathcal{U}_{j}, j=1, \ldots, r$.

We define $\mu_{0}:=0, \mu_{j}:=\sum_{i=1}^{j} \ell_{i}$,

$$
\Theta_{\ell_{j}}:=\left(\Phi_{1+\mu_{j-1}}^{j}, \ldots, \Phi_{\mu_{j}}^{j}\right)
$$

We use the following base point in $\mathrm{V}=\mathrm{V}_{\Phi} \times \mathrm{V}_{\Psi}$. For $j=1, \ldots, r$ define $\hat{\Phi}_{j}$ be the point given by

$$
\hat{\Theta}_{\ell_{j}}=\frac{-\pi}{2}\left(\left(v_{1}, \ldots, v_{\ell_{j}-1}\right), v_{\ell_{j}}\right)
$$

and $\hat{\Phi}_{i}^{j}=-\frac{\pi}{2} v_{1}$ for $i \notin\left\{\mu_{1+\mu_{j-1}}, \ldots, \mu_{j}\right\}$. In particular, $\hat{\Phi}_{\mu_{j}}^{j}=-\frac{\pi}{2} v_{\ell_{j}}$.

We now evaluate $\widetilde{P}(\widehat{\Phi}, \widehat{\Psi})$ by computing $\widetilde{P}_{j}(\widehat{\Phi}, \widehat{\Psi})$ for $j=1, \ldots, r$ :

$$
\widetilde{P}_{j}(\widehat{\Phi}, \widehat{\Psi})=i\left[\begin{array}{cccccccccc}
b_{1}^{j} & \cdots & b_{\mu_{j-1}}^{j} & b_{1+\mu_{j-1}}^{j} & b_{2+\mu_{j-1}}^{j} & \cdots & b_{\mu_{j}}^{j} & b_{\mu_{j}+1}^{j} & \cdots & b_{n}^{j} \\
b_{1}^{j} & \cdots & b_{\mu_{j-1}}^{j} & b_{1+\mu_{j-1}}^{j} & b_{2+\mu_{j-1}}^{j} & \cdots & -b_{\mu_{j}}^{j} & b_{\mu_{j}+1}^{j} & \cdots & b_{n}^{j} \\
\vdots & \cdots & \vdots & \vdots & \vdots & \cdots & \vdots & \vdots & \cdots & \vdots \\
b_{1}^{j} & \cdots & b_{\mu_{j-1}}^{j} & b_{1+\mu_{j-1}}^{j} & -b_{2+\mu_{j-1}}^{j} & \cdots & -b_{\mu_{j}}^{j} & b_{\mu_{j}+1}^{j} & \cdots & b_{n}^{j}
\end{array}\right] .
$$


Thus,

$$
\widetilde{P}(\widehat{\Phi}, \widehat{\Psi})=\left[\begin{array}{c}
\widetilde{P}_{1}(\widehat{\Phi}, \widehat{\Psi}) \\
\vdots \\
\widetilde{P}_{j}(\widehat{\Phi}, \widehat{\Psi}) \\
\vdots \\
\widetilde{P}_{r}(\widehat{\Phi}, \widehat{\Psi})
\end{array}\right]:=i \mathcal{I}_{B}
$$

where $\mathcal{I}_{B}$ is invertible by assumption. In particular, $\widetilde{P}(-\widehat{\Phi},-\widehat{\Psi})=-i \mathcal{I}_{B}$. We define

$$
\widehat{A}^{T} \equiv\left(\hat{a}_{1}, \ldots, \hat{a}_{n}\right)^{T}=\mathcal{I}_{B}^{-1} \omega^{T}
$$

which leads to the solution:

$$
F(\widehat{\Phi}, \widehat{\Psi}, \widehat{A} ; \omega)=0 .
$$

Because the $\omega_{j}$ are rationally independent, it follows that the components $\hat{a}_{k}$ of $\widehat{A}$ are all non-zero. Furthermore, it is easy to compute that

$$
J \equiv D_{(\Psi, A)} F(\widehat{\Phi}, \widehat{\Psi}, \widehat{A} ; \omega)=\left(\begin{array}{cc}
\hat{U} & i \mathcal{I}_{B} \\
\hat{U} & -i \mathcal{I}_{B}
\end{array}\right)
$$

where

$$
\hat{U}=\operatorname{diag}\left(\hat{a}_{n} b_{n}^{1} \mathcal{U}_{1}^{1}, \ldots, \hat{a}_{n} b_{n}^{r-1} \mathcal{U}_{1}^{r-1}, \hat{a}_{n} b_{n}^{r} \mathcal{U}_{\ell_{r}}^{r}\right)
$$

is a $n \times n$ matrix with diagonal blocks of dimensions $\ell_{1} \times \ell_{1}$ to $\ell_{r} \times \ell_{r}$. We compute also

$$
K \equiv D_{\Phi} F(\widehat{\Phi}, \widehat{\Psi}, \widehat{A} ; \omega)=\left(\begin{array}{c}
\hat{K} \\
\hat{K}
\end{array}\right)
$$

where

$$
\hat{K}=\operatorname{diag}\left(\hat{K}_{1}, \ldots, \hat{K}_{r}\right)
$$

is a $n \times(n-1) n$ matrix where the block $\hat{K}_{j}$ has dimensions $\ell_{j} \times(n-1) \ell_{j}$ and is of the form

$$
\begin{aligned}
& \hat{K}_{j}=\left(\begin{array}{llllll}
\hat{a}_{1} b_{1}^{j} \mathcal{U}_{1}^{j} & \cdots & \hat{a}_{1+\mu_{j-1}} b_{1+\mu_{j-1}}^{j} \mathcal{U}_{1}^{j} & \hat{a}_{2+\mu_{j-1}} b_{2+\mu_{j-1}}^{j} \mathcal{U}_{2}^{j} & \cdots & \hat{a}_{\mu_{j}} b_{\mu_{j}}^{j} \mathcal{U}_{\ell_{j}}^{j} \\
\hat{a}_{1+\mu_{j}} b_{1+\mu_{j}}^{j} \mathcal{U}_{1}^{j} & \cdots & \hat{a}_{n-1} b_{n-1}^{j} \mathcal{U}_{1}^{j}
\end{array}\right) .
\end{aligned}
$$

The matrix $J$ is invertible and its inverse is

$$
J^{-1}=\left(\begin{array}{cc}
\frac{1}{2} \hat{U}^{-1} & \frac{1}{2} \hat{U}^{-1} \\
\frac{-i}{2} \mathcal{I}_{B}^{-1} & \frac{i}{2} \mathcal{I}_{B}^{-1}
\end{array}\right) .
$$

By the implicit function theorem, there exists a neighborhood $N$ of $\widehat{\Phi}$ in $\mathrm{V}_{\Phi}$ and a unique smooth function

$$
\begin{aligned}
& G: N \longmapsto \mathrm{T} \times \mathbb{R}^{n} \\
& G: \Phi \longmapsto G(\Phi)=\left(G_{\Psi}(\Phi), G_{A}(\Phi)\right)
\end{aligned}
$$


such that

$$
G(\widehat{\Phi})=(\widehat{\Psi}, \widehat{A})
$$

and

$$
F(\Phi, G(\Phi) ; \omega) \equiv 0, \forall \Phi \in N
$$

Implicit differentiation of (42) yields that

$$
\begin{aligned}
D G(\widehat{\Phi}) & =\left(\begin{array}{c}
D G_{\Psi}(\widehat{\Phi}) \\
D G_{A}(\widehat{\Phi})
\end{array}\right)=-J^{-1} K \\
& =\left(\begin{array}{c}
\operatorname{diag}\left(\mathcal{M}_{1}, \ldots, \mathcal{M}_{r}\right) \\
*
\end{array}\right),
\end{aligned}
$$

where $*$ is not important for our purposes and the first component is a $n \times(n-1) n$ matrix composed of $n-1$ block matrices $\mathcal{M}_{j}=$

$$
\begin{aligned}
& \left(\begin{array}{ccccc}
-\frac{\hat{a}_{1} b_{1}^{j}}{\hat{a}_{\mu_{j}} b_{\mu_{j}}^{j}}\left(\mathcal{U}_{1}^{j}\right)^{2} & \ldots & -\frac{\hat{a}_{1+\mu_{j-1}} b_{1+\mu_{j-1}}^{j}}{\hat{a}_{\mu_{j}} b_{\mu_{j}}^{j}}\left(\mathcal{U}_{1}^{j}\right)^{2} & -\frac{\hat{a}_{2+\mu_{j-1}} b_{2+\mu_{j-1}}^{j}}{\hat{a}_{\mu_{j}} b_{\mu_{j}}^{j}} \mathcal{U}_{1}^{j} \mathcal{U}_{2}^{j} & \cdots \\
-\frac{\hat{a}_{\mu_{j}} b_{\mu_{j}}^{j}}{\hat{a}_{\mu_{j}} b_{\mu_{j}}^{j}} \mathcal{U}_{1}^{j} \mathcal{U}_{\ell_{j}}^{j} & -\frac{\hat{a}_{1+\mu_{j}} b_{1+\mu_{j}}^{j}}{\hat{a}_{\mu_{j}} b_{\mu_{j}}^{j}}\left(\mathcal{U}_{1}^{j}\right)^{2} & \cdots & -\frac{\hat{a}_{n-1} b_{n-1}^{j}}{\hat{a}_{\mu_{j}} b_{\mu_{j}}^{j}}\left(\mathcal{U}_{1}^{j}\right)^{2}
\end{array}\right)
\end{aligned}
$$

of dimension $\ell_{j} \times(n-1) \ell_{j}$ where $j=1, \ldots, r-1$. Recall that $\mu_{r}=n$, so that we have

$$
\begin{gathered}
\mathcal{M}_{r}=\left(\begin{array}{ccccc}
-\frac{\hat{a}_{1} b_{1}^{r}}{\hat{a}_{\mu_{r}} b_{\mu_{r}}^{r}} \mathcal{U}_{\ell_{r}}^{r} \mathcal{U}_{1}^{r} & \cdots & -\frac{\hat{a}_{\mu_{r-1}} b_{\mu_{r-1}}^{r}}{\hat{a}_{\mu_{r}} b_{\mu_{r}}^{r}} \mathcal{U}_{\ell_{r}}^{r} \mathcal{U}_{1}^{r} & -\frac{\hat{a}_{1+\mu_{r-1}} b_{1+\mu_{r-1}}^{r}}{\hat{a}_{\mu_{r}} b_{\mu_{r}}^{r}} \mathcal{U}_{\ell_{r}}^{r} \mathcal{U}_{1}^{r} \\
-\frac{\hat{a}_{2+\mu_{r-1}} b_{2+\mu_{r-1}}^{r}}{\hat{a}_{\mu_{r}} b_{\mu_{r}}^{r}} \mathcal{U}_{\ell_{r}}^{r} \mathcal{U}_{2}^{r} & \cdots & -\frac{\hat{a}_{n-1} b_{n-1}^{r}}{\hat{a}_{\mu_{r}} b_{\mu_{r}}^{r}} \mathcal{U}_{\ell_{r}}^{r} \mathcal{U}_{\ell_{r}-1}^{r}
\end{array}\right) \\
-
\end{gathered}
$$

Consequently,

$$
D G_{\Psi}(\widehat{\Phi})=\operatorname{diag}\left(\mathcal{M}_{1}, \ldots, \mathcal{M}_{r}\right)
$$

is nonsingular and it follows that the mapping

$$
G_{\Psi}: N \longrightarrow \mathrm{T}
$$

is regular at $\widehat{\Phi}$.

Consider the following $n-1$ vectors in $\left(\mathbb{R}^{\ell_{1}}\right)^{n-1} \times\left(\mathbb{R}^{\ell_{2}}\right)^{n-1} \times \cdots \times\left(\mathbb{R}^{\ell_{r}}\right)^{n-1} \simeq\left(\mathbb{R}^{n}\right)^{n-1}$ :

$$
\begin{aligned}
W_{1} & =\left(\omega^{1}, 0, \ldots, 0 ; \omega^{2}, 0, \ldots, 0 ; \ldots ; \omega^{r}, 0, \ldots, 0\right) \\
W_{2} & =\left(0, \omega^{1}, \ldots, 0 ; 0, \omega^{2}, \ldots, 0 ; \ldots, 0, \omega^{r}, \ldots, 0\right) \\
& \vdots \\
W_{n-2} & =\left(0, \ldots, \omega^{1}, 0 ; 0, \ldots, \omega^{2}, 0 ; \ldots ; 0, \ldots, \omega^{r}, 0\right) \\
W_{n-1} & =\left(0, \ldots, 0, \omega^{1} ; 0, \ldots, 0, \omega^{2} ; \ldots, 0, \ldots, 0, \omega^{r}\right),
\end{aligned}
$$


where 0 represents the 0 vector in the respective space $\mathbb{R}^{\ell_{j}}$, and we recall that

$$
\left(\omega^{1}, \ldots, \omega^{r}\right)=\left(\omega_{1}^{1}, \ldots, \omega_{\ell_{1}}^{1}, \ldots, \omega_{1}^{r}, \ldots, \omega_{\ell_{r}}^{r}\right) .
$$

The set $\left\{W_{1}, \ldots, W_{n-1}\right\}$ is linearly independent, so for any $\Phi \in \mathrm{V}_{\Phi}$, the set

$$
\mathcal{S}_{\Phi}=\left\{\Phi+\sum_{j=1}^{n-1} \sigma_{j} W_{j}\left(\bmod \mathrm{V}_{\Phi}\right)|0 \leq| \sigma_{j} \mid<<1, j=1, \ldots, n\right\}
$$

is a small $n$-1-dimensional surface through $\Phi$ in $\mathrm{V}_{\Phi}$. We are interested in showing that for $\Phi$ close enough to $\widehat{\Phi}$ in $N$, the image of $\mathcal{S}_{\Phi}$ by $G_{\Psi}$ in $\mathrm{V}_{\Psi}$ is transverse to the integral curves of the vector field $\dot{\Psi}=\omega$. To show this, we consider the function

$$
\mathcal{T}: N \longrightarrow \mathbb{R}
$$

defined by

$$
\mathcal{T}(\Phi)=\operatorname{det}\left(D G_{\Psi}(\Phi) \cdot W_{1}^{T} \quad D G_{\Psi}(\Phi) \cdot W_{2}^{T} \quad \cdots \quad D G_{\Psi}(\Phi) \cdot W_{n-1}^{T} \quad \omega^{T}\right)
$$

and recalling that $\left(\mathcal{U}_{j}^{i}\right)^{2}=I$ for all $j, i$ we compute

$$
\begin{aligned}
\mathcal{T}(\hat{\Phi}) & =\operatorname{det}\left(D G_{\Psi}(\hat{\Phi}) \cdot W_{1}^{T} \quad D G_{\Psi}(\hat{\Phi}) \cdot W_{2}^{T} \quad \cdots \quad D G_{\Psi}(\hat{\Phi}) \cdot W_{n-1}^{T} \quad \omega^{T}\right) \\
& =\operatorname{det}\left(\alpha_{j k}\right)
\end{aligned}
$$

where $j=1, \ldots, r, k=1, \ldots, n$. The elements of the matrix $\left(\alpha_{j k}\right)$ are

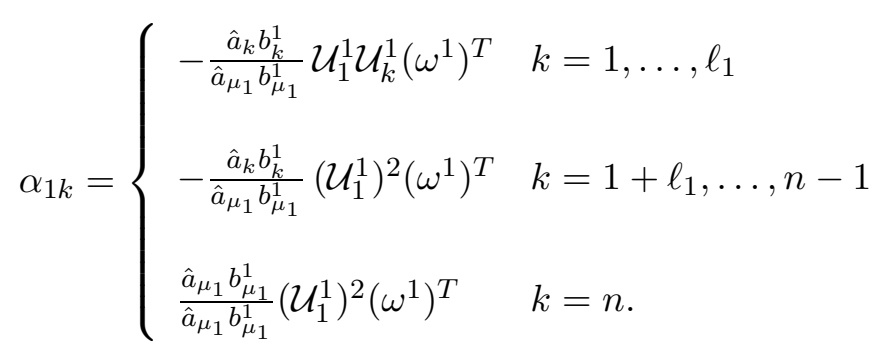

for $j=2, \ldots, r-1$

$$
\alpha_{j k}= \begin{cases}-\frac{\hat{a}_{k} b_{k}^{j}}{\hat{a}_{\mu_{j}} b_{\mu_{j}}^{j}}\left(\mathcal{U}_{1}^{j}\right)^{2}\left(\omega^{j}\right)^{T} & k=1, \ldots, \mu_{j-1} \quad \text { and } k=\mu_{j}+1, \ldots, n-1 \\ -\frac{\hat{a}_{k} b_{k}^{j}}{\hat{a}_{\mu_{j}} b_{\mu_{j}}^{j}} \mathcal{U}_{1}^{j} \mathcal{U}_{k-\mu_{j-1}}^{j}\left(\omega^{j}\right)^{T} & k=1+\mu_{j-1}, \ldots, \mu_{j} \\ \frac{\hat{a}_{\mu_{j}} b_{\mu_{j}}^{j}}{\hat{a}_{\mu_{j}} b_{\mu_{j}}^{j}}\left(\mathcal{U}_{1}^{j}\right)^{2}\left(\omega^{j}\right)^{T} & k=n .\end{cases}
$$


and finally

$$
\alpha_{r k}= \begin{cases}-\frac{\hat{a}_{k} b_{k}^{r}}{\hat{a}_{\mu_{r}} b_{\mu_{r}}^{r}} \mathcal{U}_{\ell_{r}}^{r} \mathcal{U}_{1}^{r}\left(\omega^{r}\right)^{T} & k=1, \ldots, \mu_{r-1} \\ -\frac{\hat{a}_{k} b_{k}^{r}}{\hat{a}_{\mu_{r}} b_{\mu_{r}}^{r}} \mathcal{U}_{\ell_{r}}^{r} \mathcal{U}_{k-\mu_{r-1}}^{r}\left(\omega^{r}\right)^{T} & k=1+\mu_{r-1}, \ldots, \mu_{r}-1 \\ \frac{\hat{a}_{\mu_{r}} b_{\mu_{r}}^{r}}{\hat{a}_{\mu_{r}} b_{\mu_{r}}^{r}}\left(\mathcal{U}_{\ell_{r}-1}^{r}\right)^{2}\left(\omega^{r}\right)^{T} & k=n .\end{cases}
$$

where we recall that $\mu_{r}=n$. Note that the elements of the last column are rewritten as to lead to the significant simplification of the determinant to the following form:

$$
\mathcal{T}(\hat{\Phi})=\frac{(-1)^{n-1} \omega_{1}^{1} \cdots \omega_{\ell_{1}}^{1} \cdots \omega_{1}^{r} \cdots \omega_{\ell_{r}}^{r} \hat{a}_{1} \cdots \hat{a}_{n-1}}{\left(\hat{a}_{\mu_{1}} b_{\mu_{1}}^{1}\right)^{\ell_{1}} \cdots\left(\hat{a}_{\mu_{r}} b_{\mu_{r}}^{r}\right)^{\ell_{r}}} \operatorname{det}\left(\operatorname{diag}\left(\mathcal{U}_{1}^{1}, \mathcal{U}_{1}^{2}, \ldots, \mathcal{U}_{1}^{r-1}, \mathcal{U}_{\ell_{r}}^{r}\right)\right) \operatorname{det} \mathcal{I}_{B}^{\prime}
$$

where

$$
\mathcal{I}_{B}^{\prime}=\left(\begin{array}{c}
Q_{1} \\
Q_{2} \\
\vdots \\
Q_{r}
\end{array}\right)
$$

and for $j=1, \ldots, r$

$$
Q_{j}=\left[\begin{array}{cccccccccc}
b_{1}^{j} & \cdots & b_{\mu_{j-1}}^{j} & b_{1+\mu_{j-1}}^{j} & b_{2+\mu_{j-1}}^{j} & \cdots & b_{\mu_{j}}^{j} & b_{\mu_{j}+1}^{j} & \cdots & \hat{a}_{\mu_{j}} b_{\mu_{j}}^{j} \\
b_{1}^{j} & \cdots & b_{\mu_{j-1}}^{j} & b_{1+\mu_{j-1}}^{j} & b_{2+\mu_{j-1}}^{j} & \cdots & -b_{\mu_{j}}^{j} & b_{\mu_{j}+1}^{j} & \cdots & \hat{a}_{\mu_{j}} b_{\mu_{j}}^{j} \\
\vdots & \cdots & \vdots & \vdots & \vdots & \cdots & \vdots & \vdots & \cdots & \vdots \\
b_{1}^{j} & \cdots & b_{\mu_{j-1}}^{j} & b_{1+\mu_{j-1}}^{j} & -b_{2+\mu_{j-1}}^{j} & \cdots & -b_{\mu_{j}}^{j} & b_{\mu_{j}+1}^{j} & \cdots & \hat{a}_{\mu_{j}} b_{\mu_{j}}^{j}
\end{array}\right]
$$

is a $\ell_{j} \times n$ matrix. Moreover, $\operatorname{det} \mathcal{I}_{B}^{\prime} \neq 0$ since $\operatorname{det} \mathcal{I}_{B}^{\prime}=\hat{a}_{n} \operatorname{det} \mathcal{I}_{B}$ and $\hat{a}_{n} \neq 0$. Thus $\mathcal{T}(\hat{\Phi}) \neq 0$.

It follows that there is a neighborhood $N^{\prime} \subseteq N$ in which $\mathcal{T} \neq 0$. This is equivalent to saying that for all $\Phi \in N^{\prime}$, the image of $\mathcal{S}_{\Phi}$ by $G_{\Psi}$ in $\mathrm{V}_{\Psi}$ is transverse to the integral curves of the vector field $\dot{\Psi}=\omega$.

For each $j=1, \ldots, n-1$, the integral curves of the vector field $\dot{\Phi}^{j}=\omega$ are dense in the torus T. Thus, for any $\varepsilon>0$, there is a $\tau_{j, \varepsilon}>0$ and an $s_{j, \varepsilon}>0$ such that the integral curve segment

$$
\left\{\Phi^{j}=\tau_{j} \omega(\bmod \mathrm{T}) \mid \tau_{j, \varepsilon}-s_{j, \varepsilon}<\tau_{j}<\tau_{j, \varepsilon}+s_{j, \varepsilon}\right\}
$$

is in the $\varepsilon$-ball centered on $\frac{\pi}{2} v_{j}$ in T. For $\varepsilon>0$ small enough, the surface

$$
\left\{\Phi=\left(\tau_{1} \omega, \tau_{2} \omega, \ldots, \tau_{n-1} \omega\right)\left(\bmod \mathrm{V}_{\Phi}\right) \mid \tau_{j, \varepsilon}-s_{j, \varepsilon}<\tau_{j}<\tau_{j, \varepsilon}+s_{j, \varepsilon}\right\}
$$

is contained in $N^{\prime}$ and coincides with the surface $\mathcal{S}_{\Phi^{*}}$ for

$$
\Phi^{*}=\left(\tau_{1, \varepsilon} \omega, \tau_{2, \varepsilon} \omega, \ldots, \tau_{(n-1), \varepsilon} \omega\right) \quad\left(\bmod \mathrm{V}_{\Phi}\right)
$$


Thus, by our previous result, the $n$-1-dimensional surface $G_{\Psi}\left(\mathcal{S}_{\Phi^{*}}\right)$ is transverse to the integral curves of $\dot{\Psi}=\omega$ in $\mathrm{V}_{\Psi}$. Since these integral curves are dense in $\mathrm{V}_{\Psi}$, there are infinitely many intersections with $G_{\Psi}\left(\mathcal{S}_{\Phi^{*}}\right)$ near the point $\widehat{\Psi}=G_{\Psi}(\widehat{\Phi})$.

Let $\stackrel{\circ}{\Psi} \in G_{\Psi}\left(\mathcal{S}_{\Phi^{*}}\right)$ be such an intersection point near $\widehat{\Psi}$. Then there is a $\stackrel{\circ}{n}_{n}>0$ such that

$$
\stackrel{\circ}{\Psi}=\stackrel{\circ}{\tau_{n}} \omega\left(\bmod \mathrm{V}_{\Psi}\right) \text {. }
$$

Let $\stackrel{\circ}{\Phi} \in \mathcal{S}_{\Phi^{*}}$ be such that $G_{\Psi}(\stackrel{\circ}{\Phi})=\stackrel{\circ}{\Psi}$. Then there are $\stackrel{\circ}{\tau}_{1}>0, \stackrel{\circ}{\tau}_{2}>0, \ldots, \stackrel{\circ}{\tau}_{n-1}>0$ such that

$$
\stackrel{\circ}{\Phi}=\left(\stackrel{\circ}{\tau}_{1} \omega, \stackrel{\circ}{\tau}_{2} \omega, \ldots, \stackrel{\circ}{\tau}_{n-1} \omega\right)\left(\bmod \mathrm{V}_{\Phi}\right) .
$$

It follows from (42) that $F\left(\stackrel{\circ}{\Phi}, \stackrel{\circ}{\Psi}, G_{A}(\stackrel{\circ}{\Phi}) ; \omega\right)=0$, and by construction, this corresponds to a solution of (401).

\section{Conclusion}

We have shown in this paper that $n$ nonresonant eigenvalues on the imaginary axis can be realized by a scalar delay-differential equation with $n$ delays. Moreover, the same is true for any collection of $n$ imaginary eigenvalues in a neighborhood of an $n$-tuple of nonresonant imaginary eigenvalues. We have also shown how these results can be applied to non-scalar delay-differential equations in the context of symmetric delay-differential equations where the characteristic equation decomposes according to the isotypic decomposition. We apply our result to delay-coupled $\mathbf{D}_{n}$ symmetric cell systems with $n$-odd.

There are several ways of extending the main result of our paper. One question we did not address in this paper is if $n$ nonresonant nonzero imaginary eigenvalues constitutes an upper bound for the realizability by a scalar equation with $n$ delay. The case $n=1$ is one such example since an easy calculation shows that we can have at most one imaginary eigenvalue on the imaginary axis. It is likely, but unknown, if this is also true for general $n$.

One may want to study whether $k$ zero eigenvalues in a single Jordan block and $\ell$ nonresonant nonzero imaginary eigenvalues can be realized in a scalar delay-differential equation with $k+\ell$ delays. This problem may be feasible by modifying the proof of Theorem 2.1 since the nonresonance of the $\ell$ eigenvalues is again present. However, we expect the argument used in this paper to breakdown for $n$ nonzero imaginary eigenvalues with resonance. We can also study the same problem as in this paper but for higher dimensional delay equations. One problem would be to find out if $n$ nonresonant nonzero imaginary eigenvalues can be realized by a $m$ dimensional system with $k$ delays. For instance, it is known that a pair of nonzero imaginary eigenvalues can be realized by a two-dimensional equation with one delay [8]. In this case $n=2, m=2, k=1$ and so $n=m k$; is it possible to realize three nonresonant nonzero eigenvalues or does the relationship $n=m k$ provide a bound to realizability in general?

Another problem which can be studied is whether a restriction in the class of delay equation can change the realizability. For instance, the characteristic equation for a general two-dimensional 
system with one-delay $\tau$ is

$$
\lambda^{2}+a \lambda+b \lambda e^{-\lambda \tau}+c+d e^{-\lambda \tau}=0
$$

while for a second-order equation with one delay in the feedback term we must set $b=0$. We know in this case that two nonresonant nonzero imaginary eigenvalues can be realized by a secondorder equation with a unique delay in the feedback term [4]. In fact, two imaginary eigenvalues with $1: 2$ resonance has been found in such an equation [5]. The obvious question is to see if $n$ nonresonant (and resonant) nonzero imaginary eigenvalues can be realized within the class of $n^{\text {th }}$ order scalar equations with one delay in the feedback term.

An extension of our main result in a direction relevant for studying bifurcations is to find out whether the $n$ nonresonant nonzero imaginary eigenvalues can be realized by a scalar delay equation such that the remaining eigenvalues have negative real parts; that is, the multiple Hopf point lies at the boundary of the stability region for the equilibrium solution.

Finally, let us mention the case of linear $T$-periodic equations with $N+1$ delays:

$$
\dot{x}=\sum_{j=0}^{N} a_{j}(t) x\left(t-\tau_{j}\right)
$$

where each $a_{j}(t)$ is a $T$-periodic $n \times n$ matrix. Hale [18] states the following open problem:

Is it possible to give a precise upper bound in terms of $N$ on the number of Floquet multipliers of (48) that can have moduli 1 ?

If we restrict equation (48) to scalar equations we can pose a possibly simpler problem which is related to the main result of our paper:

Is it possible to realize $N+1$ complex numbers $e^{ \pm i \omega_{1}}, \ldots, e^{ \pm i \omega_{N+1}}$ with $\omega_{1}, \ldots, \omega_{N+1}$ positive and rationally independent as Floquet multipliers of the scalar equation (48)?

This problem is automatically solved by Theorem 2.1 if a Floquet theorem can be applied to (48); that is, the Floquet exponents of the Floquet multipliers of equation (48) are eigenvalues of a scalar equation (1) with $N+1$ delays. Such a theorem has not been proved in general, however it may hold true given some conditions are imposed on (48).

\section{Acknowledgements}

This research is partly supported by the Natural Sciences and Engineering Research Council of Canada in the form of a Discovery Grant (PLB,VGL).

\section{References}

[1] J. Bélair and S.A. Campbell. Stability and bifurcations of equilibria in a multiple-delayed differential equation. SIAM J. Appl. Math. 54, (1994) 1402-1424. 
[2] A. Beuter, J. Bélair and C. Labrie. Feedback and delays in neurological diseases : a modeling study using dynamical systems. Bulletin Math. Biology 55, (1993) 525-541.

[3] A. Beuter, L. Glass, M. Mackey and M. Titcombe eds. Nonlinear Dynamics in Physiology and Medicine. Interdisciplinary Applied Mathematics, 25, Springer, New-York, 2003.

[4] S.A. Campbell, J. Bélair, T. Ohira and J. Milton. Limit cycles, tori, and complex dynamics in a second-order differential equation with delayed negative feedback. J. Dynam. Differential Equations 7 (1995), 213-236.

[5] S.A Campbell, V.G. LeBlanc. Resonant Hopf-Hopf interactions in delay differential equations. J. Dynam. Differential Equations 10 (1998), 327-346.

[6] S.A. Campbell, Y. Yuan, S. Bungay. Equivariant Hopf bifurcation in a ring of identical cells with delayed coupling, Nonlinearity 18 (2005) 2827-2846.

[7] Y. Choi and V.G. LeBlanc. Toroidal normal forms for bifurcations in retarded functional differential equations. I. Multiple Hopf and transcritical/multiple Hopf interaction. J. Differential Equations 227 (2006), 166-203.

[8] K. Cooke and Z. Grossman. Discrete delay, distributed delay and stability switches. J. Math. Anal. Appl. 86 (1982), 592-627.

[9] O. Diekmann, S.A. van Gils, S.M. Verduyn-Lunel and H.O. Walther. Delay-equations, functional, complex, and nonlinear analysis. Applied Mathematical Sciences 110, Springer-Verlag, New-York, (1995).

[10] R. Dodla, A. Sen, G.L. Johnston. Phase-locked patterns and amplitude death in a ring of delay-coupled limit cycle oscillators. Phys. Rev. E. 69 (2004), 056217.

[11] T. Faria and L.T. Magalhães. Realisation of ordinary differential equations by retarded functional differential equations in neighborhoods of equilibrium points. Proc. Roy. Soc. Ed. 125A, (1995) 759-776.

[12] L. Glass and M.C. Mackey. Oscillations and chaos in physiological control systems. Science 197, (1977) 287-289.

[13] M. Golubitsky, I. Stewart and D.G. Schaeffer. Singularities and Groups in Bifurcation Theory, Vol. II. Applied Mathematical Sciences, 69, Springer-Verlag, New York, 1988.

[14] S. Guo, Spatio-temporal patterns of nonlinear oscillations in an excitatory ring network with delay. Nonlinearity 18 (2005), 2391-2407.

[15] S. Guo, L. Huang, Hopf bifurcating periodic orbits in a ring of neurons with delays, Phys. D 183 (2003) 19-44.

[16] S. Guo and L. Huang. Stability of nonlinear waves in a ring of neurons with delays. J. Diff. Eq 236 (2007), 343-374. 
[17] M.S. Gurney, S.P. Blythe and R.M. Nisbee. Nicholson's blowflies revisited, Nature 287 (1980), $17-21$.

[18] J.K. Hale. Some problems in FDE. Fields Institute Communications 29 (2001), 195-222.

[19] J.K. Hale and S.M. Verduyn-Lunel. Introduction to functional differential equations. Applied Mathematical Sciences 99, Springer-Verlag, New-York, (1993).

[20] Y. Kuang. Delay differential equations with applications in population dynamics. Mathematics in Science and Engineering, 191. Academic Press, Boston, (1993).

[21] Y. Kuznetsov. Element of Applied Bifurcation Theory, 3rd edition. Applied Mathematical Science 112, Springer, New-York, (2004).

[22] R. Lang and K. Kobayashi. External optical feedback effects on semiconductor injection laser properties. IEEE J. Quantum Electronics 16 (1980), 347-355.

[23] M. Leite, M. Golubitsky. Homogeneous three-cell networks. Nonlinearity 19 (2006), 23132363.

[24] M. Peng. Bifurcation and stability analysis of nonlinear waves in $\mathbf{D}_{n}$-symmetric delaydifferential systems. J. Diff. Eq 232 (2007) 521-543.

[25] M. Peng, Y. Yuan, Complex dynamics in discrete delayed models with $\mathbf{D}_{4}$-symmetry. Chaos Solitons Fractals in press.

[26] B.F. Redmond, V.G. LeBlanc and A. Longtin. Bifurcation analysis of a class of first-order nonlinear delay-differential equations with reflectional symmetry. Physica D 166 (2002), 131146.

[27] E. Stone and A. Askari. Nonlinear models of chatter in drilling processes. Dyn. Syst. 17 (2002), 65-85.

[28] M.J. Suarez and P.L. Schopf. A Delayed Action Oscillator for ENSO. J. Atmos. Sci. 45 (1988), 3283-3287.

[29] J. Wu. Symmetric functional-differential equations and neural networks with memory. Trans. Amer. Math. Soc. 350 (1998), 4799-4838.

[30] J. Wu, T. Faria, Y.S. Huang. Synchronization and stable phase-locking in a network of neurons with memory. Math. Comput. Modelling 30 (1999), 117-138. 